\title{
Holographic chiral algebra: supersymmetry, infinite Ward identities, and EFTs
}

\author{
Hongliang Jiang \\ Centre for Research in String Theory, School of Physics and Astronomy, \\ Queen Mary University of London, \\ Mile End Road, E1 4 NS, U.K. \\ E-mail: h.jiang@qmul.ac.uk
}

AвSTRACT: Celestial holography promisingly reformulates the scattering amplitude holographically in terms of celestial conformal field theory living at null infinity. Recently, an infinite-dimensional symmetry algebra was discovered in Einstein-Yang-Mills theory. The starting point in the derivation is the celestial OPE of two soft currents, and the key ingredient is the summation of $\overline{\mathrm{SL}(2, \mathbb{R})}$ descendants in OPE. In this paper, we consider the supersymmetric Einstein-Yang-Mills theory and obtain the supersymmetric extension of the holographic symmetry algebra. Furthermore, we derive infinitely many Ward identities associated with the infinite soft currents which generate the holographic symmetry algebra. This is realized by considering the OPE between a soft symmetry current and a hard operator, and then summing over its $\overline{\mathrm{SL}(2, \mathbb{R})}$ descendants. These Ward identities reproduce the known Ward identities corresponding to the leading, sub-leading, and sub-sub-leading soft graviton theorems as well as the leading and sub-leading soft gluon theorems. By performing shadow transformations, we also obtain infinitely many shadow Ward identities, including the stress tensor Ward identities for sub-leading soft graviton. Finally, we use our procedure to discuss the corrections to Ward identities in effective field theory (EFT), and reproduce the corrections to soft theorems at sub-sub-leading order for graviton and sub-leading order for photon. For this aim, we derive general formulae for the celestial OPE and its corresponding Ward identities arising from a cubic interaction of three spinning massless particles. Our formalism thus provides a unified framework for understanding the Ward identities in celestial conformal field theory, or equivalently the soft theorems in scattering amplitude.

KeYwords: Conformal and W Symmetry, Conformal Field Theory, Gauge-Gravity Correspondence, Scattering Amplitudes

ArXiv EPRINT: 2108.08799 


\section{Contents}

1 Introduction 1

2 Preliminaries $\quad 4$

2.1 OPEs in supersymmetric EYM theory 4

2.2 Summing over $\overline{\mathrm{SL}(2, \mathbb{R})}$ descendants 6

$\begin{array}{lll}2.3 & \text { Mode decomposition of soft currents } & 7\end{array}$

3 Holographic chiral algebra from soft-soft OPEs 9

3.1 Pure SYM theory 9

$\begin{array}{lll}3.2 & \text { Supersymmetric EYM theory } & 10\end{array}$

$\begin{array}{lll}3.3 & \text { Structures in holography chiral algebra } & 13\end{array}$

4 Ward identities from soft-hard OPEs $\quad 14$

4.1 Graviton Ward identity 14

$\begin{array}{ll}4.2 \text { Gluon Ward identity } & 18\end{array}$

$\begin{array}{lll}4.3 & \text { Photon Ward identity } & 19\end{array}$

5 Shadow Ward identities $\quad 21$

5.1 Shadow graviton Ward identity 22

5.2 Shadow photon and gluon Ward identity 23

6 EFT corrections $\quad 24$

6.1 General celestial OPE and Ward identity 24

6.2 EFT correction to photon Ward identity 26

6.3 EFT correction to graviton Ward identity 27

6.4 Absence of EFT correction to holographic chiral algebra 28

$\begin{array}{llr}7 & \text { Conclusion } & 29\end{array}$

A Celestial super-OPEs in terms of celestial superfields 30

B Useful integrals $\quad 31$

B.1 Seed formula 31

B.2 General formula 32

B.3 Some examples 33

C General celestial OPEs in EFT 33

$\begin{array}{ll}\text { D Soft photon theorems with magnetic corrections } & 35\end{array}$ 


\section{Introduction}

Symmetry is arguably one of the most important guiding principles in physics. The content and power of symmetry have always been evolving with time. Recent studies have enriched the content of symmetry from continuous to discrete, from ordinary symmetry to higher form symmetry, and even from symmetry to non-invertible symmetry. In quantum field theory, symmetry and anomaly constrain the renormalization group flows severely. While in the swampland program, criteria regarding symmetry shed new light on quantum gravity. Undoubtedly, a better understanding of symmetry we have, a deeper aspect of fundamental physics we can acquire. In particular, symmetry enables us to establish Ward identities and conservation laws.

In this paper, we will be considering the general relativity (GR) and Yang-Mills (YM) theory as well their supersymmetrizations. Being well understood theoretically and precisely tested experimentally, GR and YM theory are the cornerstones of modern physics. In spite, the symmetry aspect remains not fully clear. For example, it was discovered more than half a century ago that asymptotic flat spacetime admits, besides the Poincare symmetries, infinite dimensional BMS symmetries [1, 2]. However, the important role of BMS symmetry was never fully appreciated until less than a decade ago due to the pioneering work $[3,4]$, where the Weinberg's soft graviton theorem was reinterpreted as the Ward identity of BMS symmetry. Since then, many interesting relations among soft theorem, memory effect and asymptotic symmetry are established. See [5] for a review. Furthermore, after many years of studies, a new holographic approach, called celestial holography, to studying quantum gravity in flat spacetime starts to emerge $[6,7]$. According to the dictionary of celestial holography, the scattering particle in the bulk spacetime can be represented as an operator $\mathcal{O}_{\Delta, J}$ in celestial conformal field theory (CCFT) living at the boundary null infinity, and the Mellin transformed scattering amplitudes, called celestial amplitudes, are just given by the conformal correlators of these celestial operators $[7,8]$. See $[9,10]$ for introductions to celestial holography.

Celestial holography seems to be a very natural language in revealing the underlying hidden symmetries of quantum theories. In this celestial approach, each non-trivial symmetry in the bulk spacetime corresponds to some soft current in CCFT. An important virtue is that this approach is free from ambiguities associated with gauge choices, boundary counter-terms, and falloff conditions, which are sometimes subtle and tedious in the direct bulk approach [11]. Another virtue is that this also makes the computations of algebra efficient due to mature techniques in 2D CFT. In particular, the soft theorems of the scattering amplitudes, which are consequences of asymptotic symmetries, are just equivalent to the Ward identities of soft currents in 2D CCFT. The soft currents are special types of celestial operators $\mathcal{O}_{\Delta, J}$ with conformal dimension $\Delta=1,0,-1, \cdots$ for bosonic fields and $\Delta=1 / 2,-1 / 2,-3 / 2, \cdots$ for fermionic fields. Operators with these special values of conformal dimension will be called soft operators, while all the rest are hard operators. So far, the Ward identities have been established at several leading orders, $\Delta=1,0,-1$ for graviton and $\Delta=1,0$ for gluon. One main goal in this paper is to derive the Ward identities associated with all the soft currents, namely for all values of $\Delta$ listed above. 
Recently, an infinite tower of symmetries was discovered for Einstein-Yang-Mills (EYM) theory in celestial holography $[11,12] .^{1}$ Each symmetry is associated with a holomorphic current, which arises from the $\overline{\mathrm{SL}(2, \mathbb{R})}$ decomposition of the soft current, and their commutators are also determined. As such, there seems to be an asymmetry between holomorphic and anti-holomorphic parts which deserves explanations. For each celestial operator and soft current, its spin $J$ in $2 \mathrm{D}$, or equivalently the helicity in $4 \mathrm{D}$, can be either positive or negative. Both positive and negative helicity soft particles generate some symmetry. However, whenever two soft particles with opposite helicity are scattered, the resulting amplitude is ambiguous and depends on the order of taking soft limits [16]. This can also be observed from the operator product expansion (OPE) of celestial operators, and ambiguities indeed arise if two operators with opposite spin are both taken soft. Therefore, there seems to be some intrinsic incompatibility between positive and negative helicity soft operators. To sidestep this subtlety, the authors in [11] focus on positive helicity soft currents only and consider CCFT with only $\operatorname{Vir} \otimes \overline{\mathrm{SL}(2, \mathbb{R})}$ symmetry, which is the subgroup of the superrotation Vir $\otimes \overline{\mathrm{Vir}}^{2}$ With $\overline{\mathrm{SL}(2, \mathbb{R})}$ global symmetry, one can decompose every positive helicity soft current into various chiral currents. To determine the commutators of these chiral currents, [11] considers the OPE of two celestial operators, which arises from the collinear limit of scattering amplitude. The important ingredient in their derivation is that one also needs to sum over all the $\overline{\mathrm{SL}(2, \mathbb{R})}$ descendants in OPEs. Taking soft limits for both positive helicity operators, which is unambiguous in this case, and decomposing the resulting soft currents into chiral currents, they obtain the desired algebra of these infinitely many chiral currents. Although such a holographic symmetry algebra only involves positive helicity soft particles, it is infinite dimensional and thus an interesting subalgebra of the full symmetry algebra in gravity and gauge theory, which remains to uncover. To emphasize the role of these chiral currents, we will also refer to this symmetry algebra as holographic chiral algebra.

In this paper, we will consider the supersymmetric Einstein-Yang-Mills theory and derive the corresponding holographic symmetry algebra by summing over the $\overline{\operatorname{SL}(2, \mathbb{R})}$ descendants of two soft currents. The resulting algebra is thus the supersymmetric extension of that in [11]. As in the case of EYM theory, we will see that the whole algebra is actually generated by the several leading order soft currents by successive commutators. As such, the new symmetries actually do not impose new extra constraints on S-matrix. This is expected from the celestial OPE as the starting point of this derivation. Indeed as shown in [17], one can bootstrap the celestial OPEs by conformal invariance as well as the soft theorems at the several leading orders.

With these infinitely many symmetries, it is then natural to ask how do these symmetries act on celestial operators and celestial amplitudes in CCFT? Furthermore, what are the corresponding Ward identities associated with these infinite symmetries? In this paper, we will show that the answers to these questions turn out to be simple: instead of considering the OPEs of two soft currents, we just need to start with the OPE between a soft current

\footnotetext{
${ }^{1}$ See also [13-15] for related discussions.

${ }^{2}$ Note that we will also work in the $(2,2)$ signature of spacetime and treat $z, \bar{z}$ as independent variables (except in section 5 and appendix B).
} 
and a hard operator. Then after further summing over the $\overline{\mathrm{SL}(2, \mathbb{R})}$ descendants similarly, we can obtain a resummed OPE between a soft current and a hard operator, which enables us to read off the action of soft current on the hard operator and establish the corresponding Ward identities. Using this approach, we establish infinitely many Ward identities for the infinite number of symmetries. In particular, we reproduce the known Ward identities corresponding to the leading, sub-leading, and sub-sub-leading soft graviton theorems as well as the leading and sub-leading soft gluon theorems. The Ward identities up to these leading orders generate all the rest of Ward identities associated with the rest of symmetries. The same method applies for fermionic symmetries associated to soft gluino and soft gravitino. We give the very explicit formulae for all the Ward identities. Importantly, we must emphasize that our Ward identities are also applicable to hard operators with negative helicities, as the celestial OPE between a soft current and a hard operator is always unambiguous, regardless of the helicities.

Furthermore, we perform the shadow transformation on the Ward identities. This gives rise to infinitely many shadow Ward identities associated with the infinite shadow symmetries. The shadow transformation seems to play an important role in celestial holography. In particular, the shadow transformation of sub-leading soft graviton current just gives the stress tensor of 2D celestial CFT, and the shadow transformation of Ward identity associated to sub-leading soft graviton is just the standard stress tensor Ward identity in 2D CFT. Our infinite shadow Ward identities are thus a straightforward generalization of this idea to all the rest of symmetries. But it remains to understand what kind of Ward identity is the most natural one in celestial holography.

Last but not least, we also make some attempts to understand how robust the holographic chiral algebra is and whether there are corrections to Ward identities. We will try to address this question in the framework of effective field theory (EFT) by inspecting the role of various higher dimensional effective field theory operators. The EFT approach to studying corrections to soft theorems was already adopted in [18]. As in [18], we will consider the cubic interactions which involve three massless particles with arbitrary helicities. We will first derive a general formula for celestial OPE arising from such a cubic vertex. This is possible as the three-point on-shell amplitude is uniquely fixed by their helicities, up to the coupling constant. With this celestial OPE, we repeat our procedure as before and obtain a general formula for Ward identities (6.5) and its shadow cousin (6.6). Applying the general result to EFT, we especially reproduce the corrections to soft theorems at sub-sub-leading order for graviton and sub-leading order for photon found in [18]. While for the holographic chiral algebra itself, we show that it is robust and free from corrections in EFT, on condition that we consider the case with only positive helicity soft particles where our formalism applies. Beyond this range of applicability, the fate of holographic chiral algebra is unknown, and a full understanding of soft negative helicity particles is required.

This paper is organized as follows. In section 2 we introduce some important preliminar-

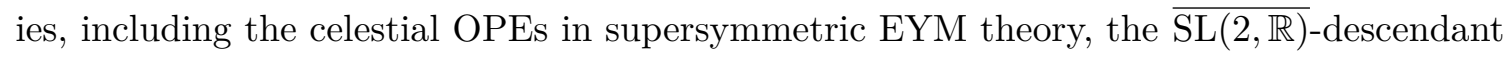
summation formula and the mode decompositions of soft currents. In section 3, we consider the soft-soft OPEs and derive the holographic chiral algebra in supersymmetric EYM theory. In section 4, we consider the soft-hard OPEs and derive infinite many Ward identities. In 
section 5, we derive the shadow Ward identities. In section 6, we apply our formalism in EFT and discuss corrections to the algebra and Ward identities. Finally, we conclude in section 7 and discuss some open questions for future research. This paper also includes four appendices. In appendix A, we rewrite the celestial OPEs in terms of celestial on-shell superfields in a compact way. In appendix B, we discuss some integrals which are vital for shadow transformation. In appendix $\mathrm{C}$, we derive a general formula for celestial $\mathrm{OPE}$ arising from a cubic interaction of three spinning massless particles. In appendix D, we review the soft photon theorems with magnetic corrections.

Note added. While we were completing the paper, we learned that the new paper [19] also obtained the celestial OPEs for arbitrary spinning operators, which is derived in our appendix $\mathrm{C}$ and subsection 6.1 .

\section{Preliminaries}

In this section, we will collect some key techniques which are very useful in the rest of the paper. As the starting point, we will first review the celestial OPEs (2.1)-(2.10) in supersymmetric EYM theory. As we discussed in the introduction, the key ingredient is to sum over all the $\overline{\mathrm{SL}(2, \mathbb{R})}$ descendant contributions in the OPE. Therefore for later convenience, we will then present a general formula (2.22) realizing this goal. Finally, we will discuss some aspects of the soft symmetry currents and particularly its decomposition into various chiral currents $(2.26)$ under $\overline{\mathrm{SL}(2, \mathbb{R})}$.

\subsection{OPEs in supersymmetric EYM theory}

Let us first review the celestial OPEs in $\mathcal{N}=1$ supersymmetric EYM theory. The OPEs can be obtained from the Mellin transformation of collinear limits of scattering amplitudes [20-22]. Alternatively, they can be bootstrapped using conformal invariance and soft theorems [17]. Especially we will be mainly focusing on the operators corresponding to positive helicity particles which are not ambiguous in the soft limit. Their explicit OPEs read: ${ }^{3}$

$$
\begin{aligned}
\mathcal{O}_{\Delta_{1},+1}^{a}\left(z_{1}, \bar{z}_{1}\right) \mathcal{O}_{\Delta_{2},+1}^{b}\left(z_{2}, \bar{z}_{2}\right) & \sim \frac{f^{a b c}}{z_{12}} B\left(\Delta_{1}-1, \Delta_{2}-1\right) \mathcal{O}_{\Delta_{1}+\Delta_{2}-1,+1}^{c}\left(z_{2}, \bar{z}_{2}\right), \\
\mathcal{O}_{\Delta_{1},+2}\left(z_{1}, \bar{z}_{1}\right) \mathcal{O}_{\Delta_{2},+2}\left(z_{2}, \bar{z}_{2}\right) & \sim-\frac{\bar{z}_{12}}{z_{12}} B\left(\Delta_{1}-1, \Delta_{2}-1\right) \mathcal{O}_{\Delta_{1}+\Delta_{2},+2}\left(z_{2}, \bar{z}_{2}\right), \\
\mathcal{O}_{\Delta_{1},+1}^{a}\left(z_{1}, \bar{z}_{1}\right) \mathcal{O}_{\Delta_{2},+2}\left(z_{2}, \bar{z}_{2}\right) & \sim-\frac{\bar{z}_{12}}{z_{12}} B\left(\Delta_{1}, \Delta_{2}-1\right) \mathcal{O}_{\Delta_{1}+\Delta_{2},+1}^{a}\left(z_{2}, \bar{z}_{2}\right), \\
\mathcal{O}_{\Delta_{1},+\frac{1}{2}}^{a}\left(z_{1}, \bar{z}_{1}\right) \mathcal{O}_{\Delta_{2},+\frac{1}{2}}^{b}\left(z_{2}, \bar{z}_{2}\right) & \sim 0 \\
\mathcal{O}_{\Delta_{1},+\frac{1}{2}}^{a}\left(z_{1}, \bar{z}_{1}\right) \mathcal{O}_{\Delta_{2},+\frac{3}{2}}\left(z_{2}, \bar{z}_{2}\right) & \sim 0, \\
\mathcal{O}_{\Delta_{1},+\frac{3}{2}}\left(z_{1}, \bar{z}_{1}\right) \mathcal{O}_{\Delta_{2},+\frac{3}{2}}\left(z_{2}, \bar{z}_{2}\right) & \sim 0 \\
\mathcal{O}_{\Delta_{1},+\frac{1}{2}}^{a}\left(z_{1}, \bar{z}_{1}\right) \mathcal{O}_{\Delta_{2},+1}^{b}\left(z_{2}, \bar{z}_{2}\right) & \sim \frac{f^{a b c}}{z_{12}} B\left(\Delta_{1}-\frac{1}{2}, \Delta_{2}-1\right) \mathcal{O}_{\Delta_{1}+\Delta_{2}-1,+\frac{1}{2}}^{c}\left(z_{2}, \bar{z}_{2}\right),
\end{aligned}
$$

\footnotetext{
${ }^{3}$ We use the convention: $f^{a b c}$ here $=-i f^{a b}{ }_{c}[11,17]$ and $\kappa_{[11,17]}=2$ here. Also for simplicity we will assume that all the particles are out-going throughout this paper.
} 


$$
\begin{aligned}
\mathcal{O}_{\Delta_{1},+\frac{1}{2}}^{a}\left(z_{1}, \bar{z}_{1}\right) \mathcal{O}_{\Delta_{2},+2}\left(z_{2}, \bar{z}_{2}\right) & \sim-\frac{\bar{z}_{12}}{z_{12}} B\left(\Delta_{1}+\frac{1}{2}, \Delta_{2}-1\right) \mathcal{O}_{\Delta_{1}+\Delta_{2},+\frac{1}{2}}^{a}\left(z_{2}, \bar{z}_{2}\right), \\
\mathcal{O}_{\Delta_{1},+1}^{a}\left(z_{1}, \bar{z}_{1}\right) \mathcal{O}_{\Delta_{2},+\frac{3}{2}}\left(z_{2}, \bar{z}_{2}\right) & \sim-\frac{\bar{z}_{12}}{z_{12}} B\left(\Delta_{1}, \Delta_{2}-\frac{1}{2}\right) \mathcal{O}_{\Delta_{1}+\Delta_{2},+\frac{1}{2}}^{a}\left(z_{2}, \bar{z}_{2}\right), \\
\mathcal{O}_{\Delta_{1},+\frac{3}{2}}\left(z_{1}, \bar{z}_{1}\right) \mathcal{O}_{\Delta_{2},+2}\left(z_{2}, \bar{z}_{2}\right) & \sim-\frac{\bar{z}_{12}}{z_{12}} B\left(\Delta_{1}-\frac{1}{2}, \Delta_{2}-1\right) \mathcal{O}_{\Delta_{1}+\Delta_{2},+\frac{3}{2}}\left(z_{2}, \bar{z}_{2}\right),
\end{aligned}
$$

where $z_{12}=z_{1}-z_{2}$ and in all the OPEs we only keep the leading singular terms, if they exist. It is worth explaining the notation a bit here. We use $\mathcal{O}_{\Delta, J}$ to denote the celestial operator with dimension $\Delta$ and spin $J$ in celestial CFT. Note that $J$ also coincides with the helicity of particles in $4 \mathrm{D}$ bulk spacetime. We also have the standard CFT relation $\Delta=h+\bar{h}, J=h-\bar{h}$ where $h$ and $\bar{h}$ are holomorphic and anti-holomorphic conformal weights, respectively. So $\mathcal{O}_{\Delta,+2}, \mathcal{O}_{\Delta,+3 / 2}$ correspond to celestial graviton and gravitino operators, while $\mathcal{O}_{\Delta,+1}^{a}, \mathcal{O}_{\Delta,+1 / 2}^{a}$ are celestial gluon and gluino operators. Here $a$ is the color index of the gauge group and $f^{a b c}$ is the corresponding anti-symmetric structure constant.

These OPEs are consistent with supersymmetry. As shown in appendix A, one can make supersymmetry manifest by introducing on-shell celestial superfields for each multiplet and then write down the corresponding super-OPEs. The supersymmetry transformation rules can be obtained easily. In particular, the supersymmetry acts on the gravity multiplet as $(\alpha, \dot{\alpha}=1,2)$

$$
\begin{aligned}
Q^{\alpha} \cdot \mathcal{O}_{\Delta,+\frac{3}{2}}(z, \bar{z}) & =z^{\alpha-1} \mathcal{O}_{\Delta+\frac{1}{2},+2}(z, \bar{z}), & & Q^{\alpha} \cdot \mathcal{O}_{\Delta,+2}(z, \bar{z})=0, \\
\tilde{Q}^{\dot{\alpha}} \cdot \mathcal{O}_{\Delta,+2}(z, \bar{z}) & =\bar{z}^{\dot{\alpha}-1} \mathcal{O}_{\Delta+\frac{1}{2},+\frac{3}{2}}(z, \bar{z}), & & \tilde{Q}^{\dot{\alpha}} \cdot \mathcal{O}_{\Delta,+\frac{3}{2}}(z, \bar{z})=0,
\end{aligned}
$$

and similarly for the vector multiplet. One can check that the OPEs above indeed transform consistently under the supersymmetry actions (2.11) and (2.12). ${ }^{4}$

For OPEs involving graviton minimally coupled matter, they actually take the following universal form with the same couplings due to the equivalence principle: ${ }^{5}$

$\mathcal{O}_{\Delta_{1}, J_{1}}\left(z_{1}, \bar{z}_{1}\right) \mathcal{O}_{\Delta_{2},+2}\left(z_{2}, \bar{z}_{2}\right) \sim-\frac{\bar{z}_{12}}{z_{12}} B\left(\Delta_{1}-J_{1}+1, \Delta_{2}-1\right) \mathcal{O}_{\Delta_{1}+\Delta_{2}, J_{1}}\left(z_{2}, \bar{z}_{2}\right), \quad J_{1}>0$.

Applying the supersymmetric transformation to (2.13), we get the general formula of OPE involving gravitino: ${ }^{6}$

$\mathcal{O}_{\Delta_{1}, J_{1}}\left(z_{1}, \bar{z}_{1}\right) \mathcal{O}_{\Delta_{2},+\frac{3}{2}}\left(z_{2}, \bar{z}_{2}\right) \sim-\frac{\bar{z}_{12}}{z_{12}} B\left(\Delta_{1}-J_{1}+1, \Delta_{2}-\frac{1}{2}\right) \mathcal{O}_{\Delta_{1}+\Delta_{2}, J_{1}-\frac{1}{2}}\left(z_{2}, \bar{z}_{2}\right), \quad J_{1} \in \mathbb{Z}_{+}$,

where $\mathcal{O}_{\Delta_{1},+J_{1}}$ should be a bosonic operator, otherwise the OPE is regular in supersymmetric EYM theory. And $\mathcal{O}_{\Delta_{1}^{\prime}, J_{1}-\frac{1}{2}}$ is the supersymmetric partner of $\mathcal{O}_{\Delta_{1}, J_{1}}$.

\footnotetext{
${ }^{4}$ Since we are considering the leading term in the OPE, it turns out to be sufficient to just consider the case $\alpha, \dot{\alpha}=1$ as $z_{1} \approx z_{2}$ to leading order. Besides, a useful identity to show SUSY invariance is $B(x, y)=B(x, y+1)+B(x+1, y)$.

${ }^{5}$ Actually this is valid even for non-positive helicity particle with $J_{1} \leq 0$ except that there may be also an extra piece which is singular in the limit $\bar{z}_{12} \rightarrow 0$.

${ }^{6}$ Again, one can consider the case $J_{1} \leq 0$ but with more complications. See [21] for explicit formulae.
} 
Before closing this subsection, let us quote some very useful formulae for Beta and Gamma functions which come from the following integral:

$$
B(x, y)=\int_{0}^{1} t^{x-1}(1-t)^{y-1} d t=\frac{\Gamma(x) \Gamma(y)}{\Gamma(x+y)} .
$$

In particular, $B(x, y)$ and $\Gamma(x)$ have simple poles at non-positive integral argument. More specifically, we have

$$
\begin{array}{rlrl}
\lim _{x \rightarrow k} \Gamma(x+m) & =\frac{1}{x-k} \frac{(-1)^{-m-k}}{(-m-k) !}, & k+m=0,-1, \cdots, \\
\lim _{x \rightarrow k} B(x+m, y) & =\frac{1}{x-k} \frac{(-1)^{-m-k}}{(-m-k) !} \frac{\Gamma(y)}{\Gamma(k+m+y)}, & k+m=0,-1, \cdots \neq y, \\
\lim _{x \rightarrow k, y \rightarrow l} B(x+m, y+l) & =\frac{x+y-k-l}{(x-k)(y-l)}\left(\begin{array}{c}
-m-n-k-l \\
-m-k
\end{array}\right), k+m, l+n=0,-1, \cdots,
\end{array}
$$

where the binomial $\left(\begin{array}{c}n \\ m\end{array}\right)=\frac{n !}{m !(n-m) !}$ and $0 !=1$.

\subsection{Summing over $\overline{\mathrm{SL}(2, \mathbb{R})}$ descendants}

One of the key ingredient in this paper is the summation of descendants in OPE which leads to OPE block. Generally the OPE of two primary operators is given by

$$
\mathcal{O}_{\Delta_{1}, J_{1}}\left(z_{1}, \bar{z}_{1}\right) \mathcal{O}_{\Delta_{2}, J_{2}}\left(z_{2}, \bar{z}_{2}\right) \sim \sum_{O_{P}} C_{\mathcal{O}_{1} \mathcal{O}_{2}}^{\mathcal{O}_{2}} \frac{\mathcal{O}_{\Delta_{P}, J_{P}}\left(z_{2}, \bar{z}_{2}\right)}{\left(z_{12} \bar{z}_{12}\right)^{\frac{\Delta_{1}+\Delta_{2}-\Delta_{P}}{2}}\left(z_{12} / \bar{z}_{12}\right)^{\frac{J_{1}+J_{2}-J_{P}}{2}}}+\cdots,
$$

where $\mathcal{O}_{\Delta_{P}, J_{P}}$ are primary operators and dots represent all the descendants. We would like to include the contributions of all the $\overline{\mathrm{SL}(2, \mathbb{R})}$ descendants. This is can be nicely realized by replacing each primary operator (together with the kinematic factors) with its corresponding OPE block. More specifically, the $\overline{\mathrm{SL}(2, \mathbb{R})}$ OPE block is given by [23]

$$
\begin{aligned}
& \overline{\mathrm{SL}(2, \mathbb{R})} \text { OPE block }=\int_{\bar{z}_{2}}^{\bar{z}_{1}} d \bar{z}_{3} \mathcal{O}_{\bar{h}_{P}}\left(\bar{z}_{3}\right)\left\langle\mathcal{O}_{\bar{h}_{1}}\left(\bar{z}_{1}\right) \mathcal{O}_{\bar{h}_{2}}\left(\bar{z}_{2}\right) \tilde{\mathcal{O}}_{1-\bar{h}_{P}}\left(\bar{z}_{3}\right)\right\rangle
\end{aligned}
$$

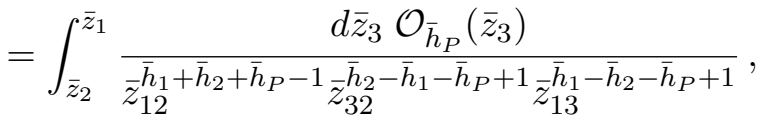

where $\tilde{\mathcal{O}}_{1-\bar{h}_{P}}=\widetilde{\mathcal{O}_{\bar{h}_{P}}}$ is the shadow of $\mathcal{O}_{\bar{h}_{P}}$ and has weight $1-\bar{h}_{P}$.

After summing over the $\overline{\mathrm{SL}(2, \mathbb{R})}$ descendants in (2.19), we then arrive at [11]

$$
\begin{aligned}
& \mathcal{O}_{\Delta_{1}, J_{1}}\left(z_{1}, \bar{z}_{1}\right) \mathcal{O}_{\Delta_{2}, J_{2}}\left(z_{2}, \bar{z}_{2}\right) \\
& \sim \sum_{O_{P}} \mathcal{N}_{\mathcal{O}_{1} \mathcal{O}_{2}}^{\mathcal{O}_{12}} \frac{\bar{z}_{12}^{N-M}}{z_{12}^{M+N}} \int_{0}^{1} d t \mathcal{O}_{\Delta_{P}, J_{P}}\left(z_{2}, \bar{z}_{2}+t \bar{z}_{12}\right) t^{\Delta_{1}-J_{1}-M+N-1}(1-t)^{\Delta_{2}-J_{2}-M+N-1},
\end{aligned}
$$

where

$$
M=\frac{\Delta_{1}+\Delta_{2}-\Delta_{P}}{2}, \quad N=\frac{J_{1}+J_{2}-J_{P}}{2},
$$


and the coefficient $\mathcal{N}_{\mathcal{O}_{1} \mathcal{O}_{2}}^{\mathcal{O}_{P}}$ can be fixed by comparing the leading term in (2.22) with (2.19):

$$
\mathcal{N}_{\mathcal{O}_{1} \mathcal{O}_{2}}^{\mathcal{O}_{2}}=\frac{C_{\mathcal{O}_{1} \mathcal{O}_{2}}^{\mathcal{O}_{2}}}{B\left(\Delta_{1}-J_{1}-M+N, \Delta_{2}-J_{2}-M+N\right)} .
$$

This formula (2.22) is the key ingredient in the rest of paper.

\subsection{Mode decomposition of soft currents}

Following the dictionary of celestial holography, the Mellin transformed scattering amplitude can be regarded as the correlator of celestial operators $\mathcal{O}_{\Delta, J}$ in CCFT. In order to form a complete basis, the dimension should reside in the principal continuous series of the unitary representations of $\mathrm{SL}(2, \mathbb{C}): \Delta \in 1+i \mathbb{R}[8]$. However, we can also analytic continue $\Delta$ in the complex plane. In particular, for special values of $\Delta$, they actually generate large gauge transformations at null infinity, and are thus the symmetry generators. Operators with these special values of dimension are called soft operators, while the rest of are called hard operators.

More specifically, for positive spin- $J$ operator, the soft symmetry currents are defined as $^{7}$

$$
R^{k, J}(z, \bar{z})=\lim _{\Delta \rightarrow k}(\Delta-k) \mathcal{O}_{\Delta,+J}(z, \bar{z}), \quad k=J, J-1, J-2, \cdots
$$

It has weights $(h, \bar{h})=\left(\frac{k+J}{2}, \frac{k-J}{2}\right)$.

These soft symmetry currents admit mode expansions under $\overline{\mathrm{SL}(2, \mathbb{R})}[11]$ :

$$
R^{k, J}(z, \bar{z})=\sum_{n=\frac{k-J}{2}}^{\frac{J-k}{2}} \frac{R_{n}^{k, J}(z)}{\bar{z}^{n+\frac{k-J}{2}}}=\bar{z}^{J-k} R_{\frac{k-J}{2}}^{k, J}(z)+\bar{z}^{J-k-1} R_{\frac{k-J+2}{2}}^{k, J}(z)+\cdots+R_{\frac{J-k}{2}}^{k, J}(z) .
$$

This gives rise to $J-k+1$ holomorphic currents $R_{n}^{k, J}(z)$ which will be referred to as chiral currents. They all have the same holomorphic weight $h=(k+J) / 2$ and transform in the $(J-k+1)$-dimensional representational of $\overline{\mathrm{SL}(2, \mathbb{R})}$.

As we will see, it turns out to be more convenient to rescale each mode and redefine the chiral currents as follows [12]:

$$
\mathcal{R}_{n}^{i, J}=(i-1-n) !(i-1+n) ! R_{n}^{J+2-2 i, J},
$$

where

$$
n=1-i, 2-i, \cdots i-1, \quad i=\frac{J-k}{2}+1=1, \frac{3}{2}, 2, \cdots .
$$

Physically, (2.27) corresponds to a light-transformation [12]. In general, the light transformations along two null directions for operator with weights $(h, \bar{h})$ in 2D CFT are given by [24]

$$
\mathbf{L}[\mathcal{O}](w, \bar{z})=\int d z(w-z)^{2 h-2} \mathcal{O}(z, \bar{z}), \quad \overline{\mathbf{L}}[\mathcal{O}](z, \bar{w})=\int d \bar{z}(\bar{w}-\bar{z})^{2 \bar{h}-2} \mathcal{O}(z, \bar{z}) .
$$

\footnotetext{
${ }^{7}$ Actually, we should exclude $k=2$ for graviton and $k=3 / 2$ for gravitino as soft currents. As we will see, they are the central terms in the algebra, and do not act on hard operators. So the honest dimension of soft currents takes values in $k=1,0,-1, \cdots$ for bosonic fields, and $k=1 / 2,-1 / 2,-3 / 2, \cdots$ for fermionic fields.
} 
Applying the second light transformation to our soft operator yields ${ }^{8}$

$$
\begin{aligned}
& \epsilon \overline{\mathbf{L}}\left[\mathcal{O}_{k+\epsilon, J}\right](z, \bar{w})=\overline{\mathbf{L}}\left[R^{k, J}\right](z, \bar{w}) \\
& =\sum_{n=\frac{k-J}{2}}^{\frac{J-k}{2}} R_{n}^{k, J}(z) \int d \bar{z}(\bar{w}-\bar{z})^{k+\epsilon-J-2} \bar{z}^{-n+\frac{J-k-\epsilon}{2}} \\
& =\sum_{n=\frac{k-J}{2}}^{\frac{J-k}{2}} R_{n}^{k, J}(z)(-2 \pi \mathrm{i}) w^{-1-n+\frac{k+\epsilon-J}{2}} \frac{\Gamma\left(n+1-\frac{k+\epsilon-J}{2}\right) \Gamma\left(1-n-\frac{k+\epsilon-J}{2}\right)}{\Gamma(2+J-k-\epsilon)} \frac{\sin \left(\pi\left(n+\frac{k+\epsilon-J}{2}\right)\right)}{\pi} .
\end{aligned}
$$

Note in the above equations, we need to deform the dimension $k$ by $\epsilon$ before applying the light transformation, but finally we need to take the limit $\epsilon \rightarrow 0$. It is easy to see that in (2.32) we can just set $\epsilon$ to 0 everywhere except for $\sin \left(\pi\left(n+\frac{k+\epsilon-J}{2}\right)\right)=(-)^{n+(k-J) / 2} \sin \frac{\pi \epsilon}{2} \approx$ $\frac{\pi \epsilon}{2}(-)^{n+\frac{k-J}{2}}$. This $\epsilon$ just cancels with $\epsilon=\Delta-k$ in the definition of soft currents. After a change of variable using (2.28), the equation (2.32) gets simplified

$$
\begin{aligned}
\overline{\mathbf{L}}\left[\mathcal{O}_{J+2-2 i, J}\right](z, \bar{z}) & =-\mathrm{i} \pi \sum_{n=1-i}^{i-1}(-)^{n+1-i} \bar{z}^{-i-n} \frac{\Gamma(i+n) \Gamma(i-n)}{\Gamma(2 i)} R_{n}^{J+2-2 i, J}(z) \\
& =\pi \mathrm{i} \frac{(-)^{2 i}}{\Gamma(2 i)} \sum_{n=1-i}^{i-1} \frac{\mathcal{R}_{n}^{i, J}}{(-\bar{z})^{i+n}}
\end{aligned}
$$

where $\mathcal{R}_{n}^{i, J}$ is precisely the same as that defined in (2.27). Therefore (2.27) is indeed equivalent to a light transformation, and $\mathcal{R}_{n}^{i, J}$ is exactly the mode expansion of lighttransformed soft operator, up to a mode-dependent sign and an overall constant. ${ }^{9}$

In order to extract each chiral currents $R_{n}^{k}(z)$ from soft symmetry currents $R^{k}(z, \bar{z})$ in (2.26), we can take derivatives for multiple times:

$$
\bar{\partial}^{p} R^{k, J}(z, \bar{z})=\sum_{n=\frac{k-J}{2}}^{\frac{J-k}{2}}\left(-n-\frac{k-J}{2}-p+1\right)_{p} \bar{z}^{-n-\frac{k-J}{2}-p} R_{n}^{k, J}(z)=p ! R_{-\frac{k-J}{2}-p}^{k, J}(z)+\mathscr{O}(\bar{z}),
$$

where $\mathscr{O}(\bar{z})$ are terms which have anti-holomorphic dependence on $\bar{z}$. Therefore, by considering holomorphic terms on the left hand side, we unambiguously select the specific chiral current $R_{-\frac{k-J}{2}-p}^{k, J}(z)$.

${ }^{8}$ Here we use the following formula to evaluate the integral

$$
\int_{-\infty}^{\infty} d \bar{z}(\bar{w}-\bar{z})^{-a} \bar{z}^{-b}=-2 \pi \mathrm{i} \frac{\bar{w}^{-a-b+1} \Gamma(a+b-1)}{\Gamma(a) \Gamma(b)}=-2 \mathrm{i} \sin (\pi b) \frac{\bar{w}^{-a-b+1} \Gamma(a+b-1) \Gamma(1-b)}{\Gamma(a)},
$$

where we evaluate the integral using Mathematica for $\operatorname{Re} a<1, \operatorname{Re} b<1, \operatorname{Re}(a+b)>1$ and then perform analytic continuation. In the second equality, we use the identity $\Gamma(x) \Gamma(1-x)=\frac{\pi}{\sin (\pi x)}$.

${ }^{9}$ Using this relation, one can infer the OPEs between hard operators and light-transformed soft operators from the OPEs between hard operators and chiral currents, which we will compute in section 4 . Alternatively, [19] directly computed the OPEs between hard operators and light-transformed soft operators. 
For redefined chiral currents in (2.27), we similarly have

$$
\bar{\partial}^{p} R^{k, J}(z, \bar{z})=p ! R_{-\frac{k-J}{2}-p}^{k, J}(z)+\mathscr{O}(\bar{z})=\frac{1}{(i-1+n) !} \mathcal{R}_{n}^{i, J}(z)+\mathscr{O}(\bar{z}),
$$

where

$$
k=J+2-2 i, \quad p=i-1-n, \quad n=\frac{J-k}{2}-p, \quad i=\frac{J-k}{2}+1 .
$$

Finally, we will use different symbols $H, I, K, L$ to label the soft currents for graviton, gravitino, gluon and gluino. As a consequence, we have the following notation:

$$
\begin{aligned}
& H^{k}=R^{k,+2}, \quad I^{k}=R^{k,+3 / 2}, \quad K^{k, a}=R^{k,+1, a}, \quad L^{k, a}=R^{k,+1 / 2, a}, \\
& \mathcal{H}_{n}^{i}=\mathcal{R}_{n}^{i,+2}, \quad \mathcal{I}_{n}^{i}=\mathcal{R}_{n}^{i,+3 / 2}, \quad \mathcal{K}_{n}^{i, a}=\mathcal{R}_{n}^{i,+1, a}, \quad \mathcal{L}_{n}^{i, a}=\mathcal{R}_{n}^{i,+1 / 2, a} .
\end{aligned}
$$

\section{Holographic chiral algebra from soft-soft OPEs}

In this section, we will derive the holographic symmetry algebra in supersymmetric EYM theory. This is realized by considering the celestial OPEs (2.1)-(2.10) where both operators are taken soft. After summing over all the $\overline{\mathrm{SL}(2, \mathbb{R})}$ descendants using $(2.22)$ and decomposing each soft current into chiral currents with (2.26), we arrive at the OPEs of chiral currents. This just yields the holographic symmetry algebra, or more precisely the holographic chiral algebra, of the chiral currents. Such an infinite-dimensional algebra is thus the underlying hidden symmetry of scattering amplitude. As we will see, these symmetries are not all independent. Instead, they are generated by several leading soft currents. Our discussion in this section is the supersymmetric generalization of [11].

We will first discuss the holographic chiral algebra in the pure SYM case which contains only gluons and gluinos. Then we will include gravitons and gravitinos and derive the full holographic chiral algebra.

\subsection{Pure SYM theory}

Let us first discuss the pure SYM theory involving gluons and gluinos only. In such a case, the OPE can be generally written as

$$
\mathcal{O}_{\Delta_{1},+J_{1}}^{a}\left(z_{1}, \bar{z}_{1}\right) \mathcal{O}_{\Delta_{2},+J_{2}}^{b}\left(z_{2}, \bar{z}_{2}\right) \sim \frac{f^{a b c}}{z_{12}} B\left(\Delta_{1}-J_{1}, \Delta_{2}-J_{2}\right) \mathcal{O}_{\Delta_{1}+\Delta_{2}-1,+\left(J_{1}+J_{2}-1\right)}^{c}\left(z_{2}, \bar{z}_{2}\right),
$$

where $J_{1}, J_{2}=1, \frac{1}{2}$ and $J_{1}+J_{2}=2, \frac{3}{2}$. Note that the OPE between two gluino operators is regular.

As described many times before, the key ingredient here is to sum over all the $\overline{\operatorname{SL}(2, \mathbb{R})}$ descendants in the OPE [11]. Using the general formula (2.22), we get

$$
\begin{aligned}
& \mathcal{O}_{\Delta_{1}, J_{1}}^{a}\left(z_{1}, \bar{z}_{1}\right) \mathcal{O}_{\Delta_{2}, J_{2}}^{b}\left(z_{2}, \bar{z}_{2}\right) \\
& \sim \frac{f^{a b c}}{z_{12}} \int_{0}^{1} d t \mathcal{O}_{\Delta_{1}+\Delta_{2}-1,+\left(J_{1}+J_{2}-1\right)}^{c}\left(z_{2}, \bar{z}_{2}+t \bar{z}_{12}\right) t^{\Delta_{1}-J_{1}-1}(1-t)^{\Delta_{2}-J_{2}-1} .
\end{aligned}
$$


Since the goal in this section is to derive the algebra of chiral currents, we thus need to separate different contributions in (2.26) for each soft symmetry current. This can be realized by taking derivatives with respect to the anti-holomorphic coordinates. Applying such derivatives to (3.2) yields

$$
\begin{aligned}
& \bar{\partial}^{p} \mathcal{O}_{\Delta_{1}, J_{1}}^{a}\left(z_{1}, \bar{z}_{1}\right) \bar{\partial}^{q} \mathcal{O}_{\Delta_{2}, J_{2}}^{b}\left(z_{2}, \bar{z}_{2}\right) \\
& \sim \frac{f^{a b c}}{z_{12}} \int_{0}^{1} d t \bar{\partial}^{p+q} \mathcal{O}_{\Delta_{1}+\Delta_{2}-1,+\left(J_{1}+J_{2}-1\right)}^{c}\left(z_{2}, \bar{z}_{2}+t \bar{z}_{12}\right) t^{\Delta_{1}-J_{1}-1+p}(1-t)^{\Delta_{2}-J_{2}-1+q} \\
& \sim \frac{f^{a b c}}{z_{12}} \sum_{s=0}^{\infty} \frac{\bar{z}_{12}^{s}}{s !} \bar{\partial}^{p+q+s} \mathcal{O}_{\Delta_{1}+\Delta_{2}-1,+\left(J_{1}+J_{2}-1\right)}^{c}\left(z_{2}, \bar{z}_{2}\right) B\left(\Delta_{1}-J_{1}+p+s, \Delta_{2}-J_{2}+q\right) .
\end{aligned}
$$

To further discuss the case of soft symmetry currents defined in (2.25), we just need to focus on special values of conformal dimension $\Delta_{1} \rightarrow k, \Delta_{2} \rightarrow l$ where $k-J_{1}, l-J_{2}=$ $0,-1,-2, \cdots$. As one can see from $(2.36)$, the leading term in $\bar{\partial}^{p} O$ is a purely holomorphic current. Therefore, in order to extract the contribution from chiral currents in (3.3), we just need to keep $s=0$ term in (3.4) which is independent of anti-holomorphic coordinates $\bar{z}_{1}, \bar{z}_{2}$. As a consequence, we find

$$
\begin{aligned}
& \bar{\partial}^{p} R^{k, J_{1}, a}\left(z_{1}, \bar{z}_{1}\right) \bar{\partial}^{q} R^{l, J_{2}, b}\left(z_{2}, \bar{z}_{2}\right) \\
& \sim \frac{f^{a b c}}{z_{12}}\left(\begin{array}{c}
-k-l+J_{1}+J_{2}-p-q \\
-k+J_{1}-p
\end{array}\right) \bar{\partial}^{p+q} R^{k+l-1, J_{1}+J_{2}-1, c}\left(z_{2}, \bar{z}_{2}\right)+\mathscr{O}\left(\bar{z}_{1}, \bar{z}_{2}\right),
\end{aligned}
$$

where we used the residue formula (2.18). Further using (2.35), we get

$$
R_{\frac{J_{1}-k}{2}-p}^{k, J_{1}, a}\left(z_{1}\right) R_{\frac{J_{2}-l}{2}-q}^{l, J_{2}, b}\left(z_{2}\right) \sim \frac{f^{a b c}}{z_{12}}\left(\begin{array}{c}
-k-l+J_{1}+J_{2}-p-q \\
-k+J_{1}-p
\end{array}\right)\left(\begin{array}{c}
p+q \\
p
\end{array}\right) R_{\frac{J_{1}+J_{2}-k-l}{2}-p-q}^{k+l, J_{1}+J_{2}-1, c}\left(z_{2}\right) .
$$

Alternatively, we can use the rescaled chiral currents defined in (2.27), which simplifies (3.6) to

$$
\mathcal{R}_{n}^{i, J_{1}, a}\left(z_{1}\right) \mathcal{R}_{m}^{j, J_{2}, b}\left(z_{2}\right) \sim \frac{f^{a b c}}{z_{12}} \mathcal{R}_{n+m}^{i+j-1, J_{1}+J_{2}-1, c}\left(z_{2}\right) .
$$

Such a dramatic simplification was one of the motivation for introducing the rescaled chiral currents in (2.27).

Spelling out the case $J_{1}, J_{2}=1,1 / 2$ explicitly and using the notation (2.39), we finally obtain

$$
\begin{aligned}
\mathcal{K}_{n}^{i, a}(z) \mathcal{K}_{m}^{j, b}(0) & \sim \frac{f^{a b c}}{z} \mathcal{K}_{n+m}^{i+j-1, c}(0), \\
\mathcal{K}_{n}^{i, a}(z) \mathcal{L}_{m}^{j, b}(0) & \sim \frac{f^{a b c}}{z} \mathcal{L}_{n+m}^{i+j-1, c}(0), \\
\mathcal{L}_{n}^{i, a}(z) \mathcal{L}_{m}^{j, b}(0) & \sim 0 .
\end{aligned}
$$

\subsection{Supersymmetric EYM theory}

Now we want to include gravity. As we show in (2.13), there is a universal OPE between graviton operator and matter operator which are minimally coupled. Just like the case of 
the SYM theory discussed above, we need to use $(2.22)$ to sum over $\overline{\operatorname{SL}(2, \mathbb{R})}$ descendants in the OPE (2.13). This then gives

$$
\mathcal{O}_{\Delta_{1}, J_{1}}\left(z_{1}, \bar{z}_{1}\right) \mathcal{O}_{\Delta_{2},+2}\left(z_{2}, \bar{z}_{2}\right) \sim-\frac{\bar{z}_{12}}{z_{12}} \int_{0}^{1} d t \mathcal{O}_{\Delta_{1}+\Delta_{2}, J_{1}}\left(z_{2}, \bar{z}_{2}+t \bar{z}_{12}\right) t^{\Delta_{1}-J_{1}}(1-t)^{\Delta_{2}-2} .
$$

Taking derivatives yields

$$
\begin{aligned}
& \bar{\partial}^{p} \mathcal{O}_{\Delta_{1}, J_{1}}\left(z_{1}, \bar{z}_{1}\right) \bar{\partial}^{q} \mathcal{O}_{\Delta_{2},+2}\left(z_{2}, \bar{z}_{2}\right) \\
& \sim-\frac{\bar{z}_{12}}{z_{12}} \sum_{s=0}^{\infty} \frac{\left(\bar{z}_{12}\right)^{s}}{s !} \bar{\partial}^{p+q+s} \mathcal{O}_{\Delta_{1}+\Delta_{2}, J_{1}}\left(z_{2}, \bar{z}_{2}\right) B\left(\Delta_{1}-J_{1}+p+s+1, \Delta_{2}-1+q\right) \\
& \quad-\frac{p}{z_{12}} \sum_{s=0}^{\infty} \frac{\left(\bar{z}_{12}\right)^{s}}{s !} \bar{\partial}^{p+q+s-1} \mathcal{O}_{\Delta_{1}+\Delta_{2}, J_{1}}\left(z_{2}, \bar{z}_{2}\right) B\left(\Delta_{1}-J_{1}+p+s, \Delta_{2}-1+q\right) \\
& \quad+\frac{q}{z_{12}} \sum_{s=0}^{\infty} \frac{\left(\bar{z}_{12}\right)^{s}}{s !} \bar{\partial}^{p+q+s-1} \mathcal{O}_{\Delta_{1}+\Delta_{2}, J_{1}}\left(z_{2}, \bar{z}_{2}\right) B\left(\Delta_{1}-J_{1}+p+s+1, \Delta_{2}-2+q\right) .
\end{aligned}
$$

Next we want to find the OPE between soft symmetry currents defined in (2.25). Just as in the case of SYM, we need to take the limit $\Delta_{1} \rightarrow k, \Delta_{2} \rightarrow l$, and only keep the $s=0$ terms in (3.13) and (3.14) which are independent of $\bar{z}_{1}$ and $\bar{z}_{2}$. As a result, we arrive at

$$
\begin{aligned}
& \bar{\partial}^{p} R^{k, J_{1}}\left(z_{1}, \bar{z}_{1}\right) \bar{\partial}^{q} R^{l,+2}\left(z_{2}, \bar{z}_{2}\right) \\
& \sim-\frac{1}{z_{12}} \frac{\left(-k-l+J_{1}-p-q+1\right) !}{\left(-k+J_{1}-p\right) !(-l+2-q) !}\left(p(2-l-q)-q\left(J_{1}-k-p\right)\right) \bar{\partial}^{p+q-1} R^{k+l, J_{1}}\left(z_{2}, \bar{z}_{2}\right)+\mathscr{O}\left(\bar{z}_{1}, \bar{z}_{2}\right),
\end{aligned}
$$

where we used the equation (2.18) and the following formula to simplify the result

$$
\begin{aligned}
& {\left[p\left(\begin{array}{c}
-k-l+J_{1}-p-q+1 \\
-k+J_{1}-p
\end{array}\right)-q\left(\begin{array}{c}
-k-l+J_{1}-p-q+1 \\
-k+J_{1}-p-1
\end{array}\right)\right]} \\
& =\frac{\left(-k-l+J_{1}-p-q+1\right) !}{\left(-k+J_{1}-p\right) !(-l+2-q) !}\left(p(2-l-q)-q\left(J_{1}-k-p\right)\right) .
\end{aligned}
$$

Using (2.36), the OPE between (redefined) chiral currents then can be straightforwardly obtained:

$$
\mathcal{R}_{n}^{i, J_{1}}\left(z_{1}\right) \mathcal{R}_{m}^{j,+2}\left(z_{2}\right) \sim-\frac{2}{z_{12}}(m(i-1)-n(j-1)) \mathcal{R}_{n+m}^{i+j-2, J_{1}}\left(z_{2}\right) .
$$

Writing out the above formula explicitly for $J_{1}=2,3 / 2,1,1 / 2$ with notation (2.39), we get the OPE between chiral graviton current and the chiral current for graviton, gravitino, gluon and gluino:

$$
\begin{aligned}
\mathcal{H}_{n}^{i}(z) \mathcal{H}_{m}^{j}(0) & \sim-\frac{2}{z}(m(i-1)-n(j-1)) \mathcal{H}_{n+m}^{i+j-2}(0), \\
\mathcal{I}_{n}^{i}(z) \mathcal{H}_{m}^{j}(0) & \sim-\frac{2}{z}(m(i-1)-n(j-1)) \mathcal{I}_{n+m}^{i+j-2}(0), \\
\mathcal{K}_{n}^{i, a}(z) \mathcal{H}_{m}^{j}(0) & \sim-\frac{2}{z}(m(i-1)-n(j-1)) \mathcal{K}_{n+m}^{i+j-2, a}(0), \\
\mathcal{L}_{n}^{i, a}(z) \mathcal{H}_{m}^{j}(0) & \sim-\frac{2}{z}(m(i-1)-n(j-1)) \mathcal{L}_{n+m}^{i+j-2, a}(0)
\end{aligned}
$$


The final non-trivial OPE we need to consider is the one between gluon and gravitino (2.9). Instead of focusing on this special OPE, we can again consider the OPE between gravitino and arbitrary bosonic operator, ${ }^{10}$ which is described in the general formula (2.14). We can then repeat the same steps for SYM and graviton to find the OPE between chiral currents. Without spelling out any details, we just write down the final result:

$$
\mathcal{R}_{n}^{i, J_{1}}\left(z_{1}\right) \mathcal{R}_{m}^{j,+\frac{3}{2}}\left(z_{2}\right) \sim-\frac{2}{z_{12}}(m(i-1)-n(j-1)) \mathcal{R}_{n+m}^{i+j-J_{1}-\frac{1}{2}}\left(z_{2}\right) .
$$

For graviton $J_{1}=2$, this agrees with (3.19). And the OPE between chiral gluon current and chiral gravitino current is given by:

$$
\mathcal{I}_{n}^{i}(z) \mathcal{K}_{m}^{j, a}(0) \sim-\frac{2}{z}(m(i-1)-n(j-1)) \mathcal{L}_{n+m}^{i+j-2, a}(0) .
$$

Finally we have two fermionic OPEs which are regular:

$$
\begin{aligned}
\mathcal{I}_{n}^{i}(z) \mathcal{I}_{m}^{j}(0) & \sim 0, \\
\mathcal{L}_{n}^{i}(z) \mathcal{I}_{m}^{j}(0) & \sim 0 .
\end{aligned}
$$

Now we obtain all the OPEs between chiral currents (3.8), (3.9), (3.10), (3.18), (3.19), (3.20), (3.21), (3.23), (3.24), (3.25). They all have at most simple poles. These OPEs can be rewritten as commutators by employing the following formula [25]:

$$
[A, B](z)=\oint_{z} \frac{d w}{2 \pi i} A(w) B(z) .
$$

Applying this formula to all the OPEs, we get the commutators between all the chiral fields. This gives rise to the holographic symmetry algebra [11]. Since all the symmetries are generated by chiral currents, we will also refer to such algebra as holographic chiral algebra. In particular, applying the formula to graviton case (3.18), we get the commutator between chiral graviton currents

$$
\left[\mathcal{H}_{n}^{i}, \mathcal{H}_{m}^{j}\right]=-2(m(i-1)-n(j-1)) \mathcal{H}_{n+m}^{i+j-2} .
$$

This turns out be just the $w_{1+\infty}$ algebra as observed in [12]. ${ }^{11}$ Note that $\mathcal{H}_{0}^{1}$ commutes with all $\mathcal{H}_{n}^{i}$ and is thus a central term. Also it is easy to see that $\mathcal{H}_{n}^{3 / 2}, \mathcal{H}_{n}^{2}, \mathcal{H}_{n}^{5 / 2}$ generate all the rest of $\mathcal{H}_{n}^{i}$ by successive commutators. Physically, this means that the algebra is actually generated by the leading, sub-leading, and sub-sub-leading soft gravitons. Similarly, in the case of gluon, the corresponding algebra is generated by the leading and sub-leading soft gluons. These are not surprising, as we start with OPEs which can actually be bootstrapped from these soft theorems [17].

The commutators for the rest of chiral currents can be obtained similarly in an obvious way as all OPEs involved have only simple poles. Furthermore, we can also consider Einstein-Maxwell theory and its supersymmetrization. The resulting algebra is almost identical except that we need to remove the color index and set $f^{a b c}$ to zero because Maxwell theory has no self-interaction.

\footnotetext{
${ }^{10}$ Note that the OPE between two fermionic operators is regular.

${ }^{11}$ To compare with [12], we need to redefine $w_{n}^{i}=\frac{1}{2} \mathcal{H}_{n}^{i}$.
} 


\subsection{Structures in holography chiral algebra}

Supersymmetry. So far, we obtained the OPEs between all chiral currents. Since we are considering the supersymmetric theory, these OPEs should be invariant under the supersymmetry transformations. Indeed, applying (2.11) (2.12) to (2.26) (2.27), one can easily derive the following supersymmetry transformation rules acting on chiral currents:

$$
\begin{array}{lll}
\mathcal{Q}^{\alpha} \cdot \mathcal{R}_{n}^{i, J}(z)=z^{\alpha-1} \mathcal{R}_{n}^{i, J+\frac{1}{2}}(z), & \mathcal{Q}^{\alpha} \cdot \mathcal{R}_{n}^{i, J+\frac{1}{2}}(z)=0, & J=\frac{3}{2}, \frac{1}{2}, \\
\tilde{\mathcal{Q}}^{\dot{\alpha}} \cdot \mathcal{R}_{n}^{i, J}(z)=(i-1-n(2 \dot{\alpha}-3)) \mathcal{R}_{n-\frac{3}{2}+\dot{\alpha}}^{i-\frac{1}{2}, J-\frac{1}{2}}(z), & \tilde{\mathcal{Q}}^{\dot{\alpha}} \cdot \mathcal{R}_{n}^{i, J+\frac{1}{2}}(z)=0, & J=2,1 .
\end{array}
$$

Then one can explicitly check that all the chiral OPEs and thus the holographic chiral algebra are indeed invariant under these SUSY transformation rules.

Generalized Sugawara construction. In the OPE of two chiral gluon current (3.8), we see that the structure is very similar to the Kac-Moody algebra at level zero, if we only keep the color indices. Furthermore, given a Kac-Moody algebra, one can then naturally construct the Sugawara stress tensor.

More specifically, we can consider the leading soft current $\mathcal{K}_{0}^{1, a}$, which is also a chiral current. It has weights $(h, \bar{h})=(1,0)$ and the OPE between $\mathcal{K}_{0}^{1, a}$ with itself just gives the Kac-Moody algebra at level zero. Therefore we can naturally the following Sugawara stress tensor:

$$
\mathcal{T}(z)=\gamma: \mathcal{K}_{0}^{1, a} \mathcal{K}_{0}^{1, a}:(z) \equiv \gamma \oint_{z} \frac{d w}{2 \pi i} \frac{1}{w-z} \mathcal{K}_{0}^{1, a}(w) \mathcal{K}_{0}^{1, a}(z),
$$

where the sum over color index $a$ should be understood, $\gamma$ is a constant to be fixed below and $: \cdots:$ is the normal order product that we defined above. Then we find

$$
\mathcal{T}(z) \mathcal{K}_{n}^{i, a}(0) \sim \frac{\mathcal{K}_{n}^{i, a}(0)}{z^{2}}+\frac{\partial \mathcal{K}_{n}^{i, a}(0)}{z}, \quad \mathcal{T}(z) \mathcal{T}(0) \sim \frac{2 \mathcal{T}(0)}{z^{2}}+\frac{\partial \mathcal{T}(0)}{z},
$$

if we choose $\gamma$ such that

$$
-\gamma f^{a b c} f^{b c d}=\delta^{a d}
$$

Hence $\gamma$ is inversely proportional to the dual Coxeter number of gauge group. Therefore $\mathcal{T}$ indeed behaves as a stress tensor in this soft sector. This Sugawara construction was considered before in $[26] .^{12}$

More generally, we also find ${ }^{13}$

$$
\begin{aligned}
& \gamma: \mathcal{K}_{m_{1}}^{j_{1}, b} \mathcal{K}_{m_{2}}^{j_{2}, b}:(z) \mathcal{K}_{n}^{i, a}(0) \sim \frac{\mathcal{K}_{n+m_{1}+m_{2}}^{i+j_{1}+j_{2}-2, a}(0)}{z^{2}}+\frac{\partial \mathcal{K}_{n+m_{1}+m_{2}}^{i+j_{1}+j_{2}-2, a}(0)}{z}, \\
& \gamma: \mathcal{K}_{m_{1}}^{j_{1}, b} \mathcal{K}_{m_{2}}^{j_{2}, b}:(z) \mathcal{L}_{n}^{i, a}(0) \sim \frac{\mathcal{L}_{n+m_{1}+m_{2}}^{i+j_{1}+2, a}(0)}{z^{2}}+\frac{\partial \mathcal{L}_{n+m_{1}+m_{2}}^{i+j_{1}+j_{2}-2, a}(0)}{z} .
\end{aligned}
$$

So it also holds if we replace $\mathcal{K}$ with $\mathcal{L}$ in (3.31). This is the generalized Sugawara construction whose physical implications remain to be understood.

\footnotetext{
${ }^{12}$ In [26], they also discussed OPE between $\mathcal{T}$ and hard operator, and found that $\mathcal{T}$ constructed in this way does not behave properly anymore.

${ }^{13}$ Here we need to use the property : $\mathcal{K}_{n}^{i, a} \mathcal{K}_{m}^{j, b}:=: \mathcal{K}_{m}^{j, a} \mathcal{K}_{n}^{i, b}$ : which follows from the mode expansion of $: \mathcal{O}_{\Delta_{1},+1}^{a} \mathcal{O}_{\Delta_{2},+1}^{b}:=: \mathcal{O}_{\Delta_{2},+1}^{a} \mathcal{O}_{\Delta_{1},+1}^{b}$, which is an operator with dimension $\Delta_{1}+\Delta_{2}$. Similarly, we also have $: \mathcal{K}_{n}^{i, a} \mathcal{L}_{m}^{j, b}:=: \mathcal{K}_{m}^{j, a} \mathcal{L}_{n}^{i, b}$ :. An extra identity is needed in the fermionic case: $\partial \mathcal{L}_{n+m}^{i+j-1, c}=-2 \gamma f^{a b c}: \mathcal{K}_{n}^{i, a} \mathcal{L}_{m}^{j, b}:$, which is just a null state relation.
} 


\section{Ward identities from soft-hard OPEs}

In the last section, we obtained the holographic chiral algebra in supersymmetric EYM theory. They are the hidden symmetry of scattering amplitude. A natural question to ask is how do these symmetries act on amplitude? And what are the physical consequences of these symmetries?

In this section, we will show that the infinite number of soft symmetry currents just lead to an infinite number of Ward identities. Each Ward identity just relates celestial amplitudes with and without the insertion of soft current. The way to derive these Ward identities is almost identical to the derivation of symmetry algebra in the last section, except that we need to use the soft-hard OPE now. More specifically, we will pick the celestial OPEs (2.1)-(2.10) and take one of them to be soft. After summing over all the $\overline{\mathrm{SL}(2, \mathbb{R})}$ descendants, we will arrive at the OPE between soft currents and hard operators. It turns out that this just yields the Ward identity of the corresponding soft current. If we further decompose the soft current into chiral currents using (2.26), we arrive at the chiral Ward identities for the chiral currents.

These Ward identities reproduce the known Ward identities corresponding to the leading, sub-leading and sub-sub-leading soft graviton theorems as well as the leading and sub-leading soft gluon theorems. Since the whole tower of soft currents is generated by these several leading soft currents, the resulting tower of Ward identities is thus also generated by the Ward identities of these several leading order soft currents. Nevertheless, our formulae for all the Ward identities are explicit and may shed new light on the structure of holographic chiral algebra. This method also works for fermionic symmetry current, but we will not discuss their corresponding Ward identities explicitly in this section. Instead, we will present a general OPE formula (6.1) from which a general formula for Ward identities (6.5) is derived. Specializing the general formula (6.5) to soft gluino or soft gravitino, one easily obtains their corresponding Ward identities.

\subsection{Graviton Ward identity}

As we discussed, our basic strategy is to consider the OPE with all $\overline{\mathrm{SL}(2, \mathbb{R})}$ descendants included, and then specialize to the case of soft-hard OPE. Let us first discuss the case of graviton. The general OPE involving graviton after summing over $\overline{\mathrm{SL}(2, \mathbb{R})}$ descendants is given in (3.11):

$$
\mathcal{O}_{\Delta_{1},+2}\left(z_{1}, \bar{z}_{1}\right) \mathcal{O}_{\Delta_{2}, J_{2}}\left(z_{2}, \bar{z}_{2}\right) \sim-\frac{\bar{z}_{12}}{z_{12}} \int_{0}^{1} d t \mathcal{O}_{\Delta_{1}+\Delta_{2}, J_{2}}\left(z_{2}, \bar{z}_{2}+t \bar{z}_{12}\right) t^{\Delta_{1}-2}(1-t)^{\Delta_{2}-J_{2}}
$$

After performing the integral on the right hand side, we get

$$
\begin{aligned}
& \mathcal{O}_{\Delta_{1},+2}\left(z_{1}, \bar{z}_{1}\right) \mathcal{O}_{\Delta_{2}, J_{2}}\left(z_{2}, \bar{z}_{2}\right) \\
& \sim-\frac{\bar{z}_{12}}{z_{12}} \sum_{s=0}^{\infty} \frac{\left(\bar{z}_{12}\right)^{s}}{s !} \partial^{s} \mathcal{O}_{\Delta_{1}+\Delta_{2},+J_{2}}\left(z_{2}, \bar{z}_{2}\right) B\left(\Delta_{1}+s-1, \Delta_{2}-J_{2}+1\right) .
\end{aligned}
$$


We need to consider the soft graviton for $\mathcal{O}_{\Delta_{1},+2}$, so we set $\Delta_{1} \rightarrow k$. Then the above OPE simplified as follows:

$$
\begin{aligned}
& H^{k}\left(z_{1}, \bar{z}_{1}\right) \mathcal{O}_{\Delta_{2}, J_{2}}\left(z_{2}, \bar{z}_{2}\right) \\
& \sim-\frac{\bar{z}_{12}}{z_{12}} \sum_{s=0}^{1-k} \frac{\left(\bar{z}_{12}\right)^{s}}{s !} \bar{\partial}^{s} \mathcal{O}_{\Delta_{2}+k,+J_{2}}\left(z_{2}, \bar{z}_{2}\right) \frac{(-1)^{-s+1-k}}{(-s+1-k) !} \frac{\Gamma\left(\Delta_{2}-J_{2}+1\right)}{\Gamma\left(\Delta_{2}-J_{2}+k+s\right)},
\end{aligned}
$$

where we used the formula (2.17) and the infinite sum of $s$ truncates because we need a pole from $\Gamma\left(\Delta_{1}+s-1\right)$. This is the OPE between the soft graviton current and a hard operator.

We also want to find the OPE between chiral currents and the hard operators. For this purpose, we expand $\left(\bar{z}_{1}-\bar{z}_{2}\right)^{s+1}$ :

$$
\begin{aligned}
& H^{k}\left(z_{1}, \bar{z}_{1}\right) \mathcal{O}_{\Delta_{2}, J_{2}}\left(z_{2}, \bar{z}_{2}\right) \\
& \sim-\frac{1}{z_{12}} \sum_{s=0}^{1-k} \sum_{n=0}^{s+1}\left(\begin{array}{c}
s+1 \\
n
\end{array}\right) \frac{\bar{z}_{1}^{n}\left(-\bar{z}_{2}\right)^{s+1-n}}{s !} \bar{\partial}^{s} \mathcal{O}_{\Delta_{2}+k,+J_{2}}\left(z_{2}, \bar{z}_{2}\right) \frac{(-1)^{-s+1-k}}{(-s+1-k) !} \frac{\Gamma\left(\Delta_{2}-J_{2}+1\right)}{\Gamma\left(\Delta_{2}-J_{2}+k+s\right)} \\
& \sim-\frac{1}{z_{12}} \sum_{n=0}^{2-k} \sum_{s=\max (0, n-1)}^{1-k}\left(\begin{array}{c}
s+1 \\
n
\end{array}\right) \bar{z}_{1}^{n} \bar{z}_{2}^{s+1-n} \bar{\partial}^{s} \mathcal{O}_{\Delta_{2}+k,+J_{2}}\left(z_{2}, \bar{z}_{2}\right) \frac{(-1)^{-n-k}}{s !(-s+1-k) !} \frac{\Gamma\left(\Delta_{2}-J_{2}+1\right)}{\Gamma\left(\Delta_{2}-J_{2}+k+s\right)} .
\end{aligned}
$$

Note the non-trivial exchange of sums over $s$ and $n$. On the other hand, the soft current can also be expanded in terms of chiral currents using (2.26) and (2.27). Inserting the expansion into (4.5) and comparing the left and right hand sides, we find the following OPE

$$
\begin{aligned}
& \mathcal{H}_{n}^{i}\left(z_{1}\right) \mathcal{O}_{\Delta_{2}, J_{2}}\left(z_{2}, \bar{z}_{2}\right) \sim \frac{(-1)^{n+i}}{z_{12}} \sum_{r=\max (0, n-i+2)}^{n+i-1}\left(\begin{array}{c}
i+n-1 \\
r
\end{array}\right) \frac{(r-n+i-1) \Gamma\left(\Delta_{2}-J_{2}+1\right)}{\Gamma\left(\Delta_{2}-J_{2}+r-n-i+2\right)} \\
& \times \bar{z}_{2}^{r} \bar{\partial}^{r-n+i-2} \mathcal{O}_{\Delta_{2}-2 i+4, J_{2}}\left(z_{2}, \bar{z}_{2}\right) .
\end{aligned}
$$

Spelling out the OPE explicitly at several leading orders, we get $^{14}$

- leading soft graviton $\left(\Delta_{H}=1\right)$ :

$$
\begin{aligned}
\mathcal{H}_{1 / 2}^{3 / 2}\left(z_{1}\right) \mathcal{O}_{\Delta,, J}\left(z_{2}, \bar{z}_{2}\right) & \sim \frac{\bar{z}_{2}}{z_{12}} \mathcal{O}_{\Delta+1, J}\left(z_{2}, \bar{z}_{2}\right), \\
\mathcal{H}_{-1 / 2}^{3 / 2}\left(z_{1}\right) \mathcal{O}_{\Delta, J}\left(z_{2}, \bar{z}_{2}\right) & \sim \frac{-1}{z_{12}} \mathcal{O}_{\Delta+1, J}\left(z_{2}, \bar{z}_{2}\right),
\end{aligned}
$$

- sub-leading soft graviton $\left(\Delta_{H}=0\right)$ :

$$
\begin{aligned}
\frac{1}{2} \mathcal{H}_{1}^{2}\left(z_{1}\right) \mathcal{O}_{\Delta, J}\left(z_{2}, \bar{z}_{2}\right) & \sim-\frac{\bar{z}_{2}^{2} \partial_{\bar{z}_{2}}+2 \bar{h} \bar{z}_{2}}{z_{12}} \mathcal{O}_{\Delta, J}\left(z_{2}, \bar{z}_{2}\right), \\
\mathcal{H}_{0}^{2}\left(z_{1}\right) \mathcal{O}_{\Delta, J}\left(z_{2}, \bar{z}_{2}\right) & \sim 2 \frac{\bar{z}_{2} \partial_{\bar{z}_{2}}+\bar{h}}{z_{12}} \mathcal{O}_{\Delta, J}\left(z_{2}, \bar{z}_{2}\right), \\
\frac{1}{2} \mathcal{H}_{-1}^{2}\left(z_{1}\right) \mathcal{O}_{\Delta, J}\left(z_{2}, \bar{z}_{2}\right) & \sim-\frac{\partial_{\bar{z}_{2}}}{z_{12}} \mathcal{O}_{\Delta, J}\left(z_{2}, \bar{z}_{2}\right),
\end{aligned}
$$

\footnotetext{
${ }^{14}$ Note $2 \bar{h}=\Delta-J$. Also $\mathcal{H}_{0}^{1}$ is a central term and acts on hard operators trivially as one can see from (4.4) by setting $k \rightarrow 2$.
} 
- sub-sub-leading soft graviton $\left(\Delta_{H}=-1\right)$ :

$$
\begin{aligned}
\frac{1}{6} \mathcal{H}_{\frac{3}{2}}^{\frac{5}{2}}(w) \mathcal{O}_{\Delta, J}(z, \bar{z}) & \sim \frac{\bar{z}^{3} \partial_{\bar{z}}^{2}+4 \bar{h} \bar{z}^{2} \partial_{\bar{z}}+2 \bar{h}(2 \bar{h}-1) \bar{z}}{2(w-z)} \mathcal{O}_{\Delta-1, J}(z, \bar{z}) \\
\frac{1}{2} \mathcal{H}_{\frac{1}{2}}^{\frac{5}{2}}(w) \mathcal{O}_{\Delta, J}(z, \bar{z}) & \sim-\frac{3 \bar{z}^{2} \partial_{\bar{z}}^{2}+8 \bar{h} \bar{z} \partial_{\bar{z}}+2 \bar{h}(2 \bar{h}-1)}{2(w-z)} \mathcal{O}_{\Delta-1, J}(z, \bar{z}) \\
\frac{1}{2} \mathcal{H}_{-\frac{1}{2}}^{\frac{5}{2}}(w) \mathcal{O}_{\Delta, J}(z, \bar{z}) & \sim \frac{3 \bar{z} \partial_{\bar{z}}^{2}+4 \bar{h} \partial_{\bar{z}}}{2(w-z)} \mathcal{O}_{\Delta-1, J}(z, \bar{z}) \\
\frac{1}{6} \mathcal{H}_{-\frac{3}{2}}^{\frac{5}{2}}(w) \mathcal{O}_{\Delta, J}(z, \bar{z}) & \sim-\frac{\partial_{\bar{z}}^{2}}{2(w-z)} \mathcal{O}_{\Delta-1, J}(z, \bar{z})
\end{aligned}
$$

where the coefficients on the left hand side of OPE is just the rescaling factor in (2.27).

The OPEs (4.5), (4.7) as well as the explicit forms in the several leading orders just give the transformation rules of hard operators under the action soft symmetry current and chiral current. Up to sub-sub-leading order, it turns out the structures here are identical to that in $[13,17,27]$.

Using the OPEs (4.5), (4.7) in correlators, we claim that we have the following Ward identities for soft symmetry currents

$$
\begin{aligned}
& \left\langle H^{l}(z, \bar{z}) \mathcal{O}_{\Delta_{1}, J_{1}}\left(z_{1}, \bar{z}_{1}\right) \cdots \mathcal{O}_{\Delta_{m}, J_{m}}\left(z_{m}, \bar{z}_{m}\right)\right\rangle \\
& =\sum_{k=1}^{m} \sum_{s=0}^{1-l} \frac{\left(\bar{z}-\bar{z}_{k}\right)^{s+1}}{z-z_{k}} \frac{(-1)^{-s-l}}{s !(-s+1-l) !} \frac{\Gamma\left(2 \bar{h}_{k}+1\right)}{\Gamma\left(2 \bar{h}_{k}+l+s\right)} \\
& \quad \times \bar{\partial}_{k}^{s}\left\langle\mathcal{O}_{\Delta_{1}, J_{1}}\left(z_{1}, \bar{z}_{1}\right) \cdots \mathcal{O}_{\Delta_{k}+l, J_{k}}\left(z_{k}, \bar{z}_{k}\right) \cdots \mathcal{O}_{\Delta_{m}, J_{m}}\left(z_{m}, \bar{z}_{m}\right)\right\rangle
\end{aligned}
$$

and furthermore the chiral Ward identities for chiral currents:

$$
\begin{aligned}
& \left\langle\mathcal{H}_{n}^{i}(z) \mathcal{O}_{\Delta_{1}, J_{1}}\left(z_{1}, \bar{z}_{1}\right) \cdots \mathcal{O}_{\Delta_{m}, J_{m}}\left(z_{m}, \bar{z}_{m}\right)\right\rangle \\
& =\sum_{k=1}^{m} \frac{(-1)^{n+i}}{z-z_{k}} \sum_{r=\max (0, n-i+2)}^{n+i-1}\left(\begin{array}{c}
i+n-1 \\
r
\end{array}\right) \frac{(r-n+i-1) \Gamma\left(2 \bar{h}_{k}+1\right)}{\Gamma\left(2 \bar{h}_{k}+r-n-i+2\right)} \\
& \quad \times \bar{z}_{k}^{r} \bar{\partial}_{k}^{r-n+i-2}\left\langle\mathcal{O}_{\Delta_{1}, J_{1}}\left(z_{1}, \bar{z}_{1}\right) \cdots \mathcal{O}_{\Delta_{k}-2 i+4, J_{k}}\left(z_{k}, \bar{z}_{k}\right) \cdots \mathcal{O}_{\Delta_{m}, J_{m}}\left(z_{m}, \bar{z}_{m}\right)\right\rangle,
\end{aligned}
$$

where $\bar{\partial}_{k}=\frac{\partial}{\partial \bar{z}_{k}}$.

This is not surprising: in the standard stress tensor Ward identity we essentially also replace every pair of primary operator and stress tensor with their corresponding singular OPEs. This seems to be a general feature for all Ward identities arising from symmetry in CFT. Since here all the currents also correspond to some symmetries, similar tricks should also work. Actually, in the present case, these Ward identities can be further justified as follows. Note that in OPEs (4.5), (4.7), all the $\overline{\mathrm{SL}(2, \mathbb{R})}$ descendants have been summed over, implying that the anti-holomorphic dependence is supposed to be exact in the above Ward identities. Therefore, we only need to worry about the holomorphic dependence because we have not considered the $\operatorname{SL}(2, \mathbb{R})$ descendants. However, there is a big simplification in the present case: all the OPEs (4.5), (4.7) have only simple poles in the holomorphic coordinates. If we denote the correlator on the left-hand side of Ward identity as $F(z)$, then 
we know that such a function can only be singular when $z$ hits other operator insertions $z_{i}$, and the singular behavior in the coincident limit is dictated by OPEs. Since all the OPEs here have only simple poles, we thus learn that $F(z)$ is a meromorphic function with only simples poles (note the $F(z)$ should be finite at infinity). Following the Mittag-Leffler theorem, such a meromorphic function is uniquely determined by its poles and the residues there, up to a constant. Our Ward identities have exactly the expected behavior as a meromorphic function and the undetermined constant can be argued to be zero by cluster decomposition. This thus establishes our Ward identities.

One can check explicitly that up to sub-sub-leading order, our Ward identities reproduce all the known Ward identities corresponding to leading, sub-leading and sub-sub-leading soft theorems [27-29]:

$$
\begin{aligned}
& \left\langle H^{1}(z, \bar{z}) \mathcal{O}_{\Delta_{1}, J_{1}}\left(z_{1}, \bar{z}_{1}\right) \cdots \mathcal{O}_{\Delta_{m}, J_{m}}\left(z_{m}, \bar{z}_{m}\right)\right\rangle \\
& =-\sum_{k=1}^{m} \frac{\bar{z}-\bar{z}_{k}}{z-z_{k}}\left\langle\mathcal{O}_{\Delta_{1}, J_{1}}\left(z_{1}, \bar{z}_{1}\right) \cdots \mathcal{O}_{\Delta_{k}+1,+J_{k}}\left(z_{k}, \bar{z}_{k}\right) \cdots \mathcal{O}_{\Delta_{m}, J_{m}}\left(z_{m}, \bar{z}_{m}\right)\right\rangle,
\end{aligned}
$$

and

$$
\begin{aligned}
& \left\langle H^{0}(z, \bar{z}) \mathcal{O}_{\Delta_{1}, J_{1}}\left(z_{1}, \bar{z}_{1}\right) \cdots \mathcal{O}_{\Delta_{m}, J_{m}}\left(z_{m}, \bar{z}_{m}\right)\right\rangle \\
& =\sum_{k=1}^{m} \frac{\left(\bar{z}-\bar{z}_{k}\right)^{2}}{z-z_{k}}\left[\frac{2 \bar{h}_{k}}{\bar{z}-\bar{z}_{k}}-\bar{\partial}_{k}\right]\left\langle\mathcal{O}_{\Delta_{1}, J_{1}}\left(z_{1}, \bar{z}_{1}\right) \cdots \mathcal{O}_{\Delta_{m}, J_{m}}\left(z_{m}, \bar{z}_{m}\right)\right\rangle,
\end{aligned}
$$

and

$$
\begin{aligned}
& \left\langle H^{-1}(z, \bar{z}) \mathcal{O}_{\Delta_{1}, J_{1}}\left(z_{1}, \bar{z}_{1}\right) \cdots \mathcal{O}_{\Delta_{m}, J_{m}}\left(z_{m}, \bar{z}_{m}\right)\right\rangle \\
& =-\frac{1}{2} \sum_{k=1}^{m} \frac{\left(\bar{z}-\bar{z}_{k}\right)^{3}}{z-z_{k}}\left[\frac{2 \bar{h}_{k}\left(2 \bar{h}_{k}-1\right)}{\left(\bar{z}-\bar{z}_{k}\right)^{2}}-\frac{4 \bar{h}_{k} \bar{\partial}_{k}}{\bar{z}-\bar{z}_{k}}+\bar{\partial}_{k}^{2}\right] \\
& \quad \times\left\langle\mathcal{O}_{\Delta_{1}, J_{1}}\left(z_{1}, \bar{z}_{1}\right) \cdots \mathcal{O}_{\Delta_{k}-1, J_{k}}\left(z_{k}, \bar{z}_{k}\right) \cdots \mathcal{O}_{\Delta_{m}, J_{m}}\left(z_{m}, \bar{z}_{m}\right)\right\rangle .
\end{aligned}
$$

The rest of Ward identities are supposed to be guaranteed by the associativity of the holographic chiral algebra that we discussed in the previous section.

One important remark is that our Ward identities (4.17)(4.18) hold for both positive and negative helicity hard operators, although we only considered the positive helicity soft operators in the discussion of holographic chiral algebra in the previous section. For example, the OPE of two gravitons with opposite helicity is [17]

$$
\begin{aligned}
\mathcal{O}_{\Delta_{1},+2}\left(z_{1}, \bar{z}_{1}\right) \mathcal{O}_{\Delta_{2},-2}\left(z_{2}, \bar{z}_{2}\right) \sim & -\frac{\bar{z}_{12}}{z_{12}} B\left(\Delta_{1}-1, \Delta_{2}+3\right) \mathcal{O}_{\Delta_{1}+\Delta_{2},-2}\left(z_{2}, \bar{z}_{2}\right) \\
& -\frac{z_{12}}{\bar{z}_{12}} B\left(\Delta_{1}+3, \Delta_{2}-1\right) \mathcal{O}_{\Delta_{1}+\Delta_{2},+2}\left(z_{2}, \bar{z}_{2}\right) .
\end{aligned}
$$

The first term has been considered in (4.1), so we only need to worry about the second term. The second term has a zero, instead of a pole, in the holomorphic coincident limit $z_{1} \rightarrow z_{2}$. Even at sub-sub-leading order $\Delta_{1} \rightarrow k=1,0,-1$, the second term has no contribution in soft-hard OPE because $\left(\Delta_{1}-k\right) B\left(\Delta_{1}+3, \Delta_{2}-1\right)$ vanishes at these orders. As a result, the Ward identities can not be modified up to these orders. Since we know soft gravitons up to sub-sub-leading order generate the whole tower of symmetry, the second term is supposed to have no effect either for the rest of Ward identities. 


\subsection{Gluon Ward identity}

Now we switch to the gluon case. We start with the following gluon OPE (3.2) where all the $\overline{\mathrm{SL}(2, \mathbb{R})}$ descendants have been included:

$$
\mathcal{O}_{\Delta_{1},+1}^{a}\left(z_{1}, \bar{z}_{1}\right) \mathcal{O}_{\Delta_{2}, J_{2}}^{b}\left(z_{2}, \bar{z}_{2}\right) \sim \frac{f^{a b c}}{z_{12}} \int_{0}^{1} d t \mathcal{O}_{\Delta_{1}+\Delta_{2}-1, J_{2}}\left(z_{2}, \bar{z}_{2}+t \bar{z}_{12}\right) t^{\Delta_{1}-2}(1-t)^{\Delta_{2}-J_{2}-1} .
$$

Doing the integral on the right hand side gives:

$$
\mathcal{O}_{\Delta_{1},+1}^{a}\left(z_{1}, \bar{z}_{1}\right) \mathcal{O}_{\Delta_{2}, J_{2}}^{b}\left(z_{2}, \bar{z}_{2}\right) \sim \frac{f^{a b c}}{z_{12}} \sum_{s=0}^{\infty} \frac{\left(\bar{z}_{12}\right)^{s}}{s !} \bar{\partial}^{s} \mathcal{O}_{\Delta_{1}+\Delta_{2}-1, J_{2}}\left(z_{2}, \bar{z}_{2}\right) B\left(\Delta_{1}+s-1, \Delta_{2}-J_{2}\right) .
$$

We then take $\mathcal{O}_{\Delta_{1},+1}^{a}$ soft by setting $\Delta_{1} \rightarrow k$. Then the above OPE reduces to

$$
\begin{aligned}
& K^{k, a}\left(z_{1}, \bar{z}_{1}\right) \mathcal{O}_{\Delta_{2}, J_{2}}^{b}\left(z_{2}, \bar{z}_{2}\right) \\
& \sim \frac{f^{a b c}}{z_{12}} \sum_{s=0}^{1-k} \frac{\left(\bar{z}_{12}\right)^{s}}{s !} \bar{\partial}^{s} \mathcal{O}_{\Delta_{2}+k-1, J_{2}}\left(z_{2}, \bar{z}_{2}\right) \frac{(-1)^{-s+1-k}}{(-s+1-k) !} \frac{\Gamma\left(\Delta_{2}-J_{2}\right)}{\Gamma\left(\Delta_{2}-J_{2}+k+s-1\right)},
\end{aligned}
$$

where we used (2.17) and the infinite sum of $s$ truncates because we need a pole from $\Gamma\left(\Delta_{1}+s-1\right)$.

To find the OPE between chiral gluon currents and the hard operators, we expand $\left(\bar{z}_{1}-\bar{z}_{2}\right)^{s}$ :

$$
\begin{aligned}
& K^{k, a}\left(z_{1}, \bar{z}_{1}\right) \mathcal{O}_{\Delta_{2}, J_{2}}^{b}\left(z_{2}, \bar{z}_{2}\right) \\
& \sim \frac{f^{a b c}}{z_{12}} \sum_{s=0}^{1-k} \sum_{n=0}^{s}\left(\begin{array}{l}
s \\
n
\end{array}\right) \frac{\bar{z}_{1}^{n}\left(-\bar{z}_{2}\right)^{s-n}}{s !} \bar{\partial}^{s} \mathcal{O}_{\Delta_{2}+k-1, J_{2}}\left(z_{2}, \bar{z}_{2}\right) \frac{(-1)^{-s+1-k}}{(-s+1-k) !} \frac{\Gamma\left(\Delta_{2}-J_{2}\right)}{\Gamma\left(\Delta_{2}-J_{2}+k+s-1\right)} \\
& \sim \frac{f^{a b c}}{z_{12}} \sum_{n=0}^{1-k} \sum_{s=n}^{1-k}\left(\begin{array}{l}
s \\
n
\end{array}\right) \frac{\bar{z}_{1}^{n}\left(-\bar{z}_{2}\right)^{s-n}}{s !} \bar{\partial}^{s} \mathcal{O}_{\Delta_{2}+k-1, J_{2}}\left(z_{2}, \bar{z}_{2}\right) \frac{(-1)^{-s+1-k}}{(-s+1-k) !} \frac{\Gamma\left(\Delta_{2}-J_{2}\right)}{\Gamma\left(\Delta_{2}-J_{2}+k+s-1\right)},
\end{aligned}
$$

where we exchange the sum of $s$ and $n$.

Further inserting the mode expansion into the above OPE and comparing the left and right hand sides, we find the following OPE between chiral current and hard operator

$$
\begin{aligned}
& \mathcal{K}_{n}^{i, a}\left(z_{1}\right) \mathcal{O}_{\Delta_{2}, J_{2}}^{b}\left(z_{2}, \bar{z}_{2}\right) \\
& \sim \frac{f^{a b c}}{z_{12}} \sum_{r=0}^{i-1+n}(-1)^{i-1+n}\left(\begin{array}{c}
i-1+n \\
r
\end{array}\right) \bar{z}_{2}^{r} \bar{\partial}^{r+i-1-n} \mathcal{O}_{\Delta_{2}+2-2 i, J_{2}}\left(z_{2}, \bar{z}_{2}\right) \frac{\Gamma\left(\Delta_{2}-J_{2}\right)}{\Gamma\left(\Delta_{2}-J_{2}+1-i-n+r\right)} .
\end{aligned}
$$

At first two leading orders, the OPE explicitly reads:

- leading soft gluon $\left(\Delta_{K}=1\right)$ :

$$
\mathcal{K}_{0}^{1, a}(z) \mathcal{O}_{\Delta, J}^{b}(w, \bar{w}) \sim \frac{f^{a b c}}{z-w} \mathcal{O}_{\Delta, J}^{c}(w, \bar{w}),
$$


- sub-leading soft gluon $\left(\Delta_{K}=0\right)$ :

$$
\begin{aligned}
\mathcal{K}_{\frac{1}{2}}^{\frac{3}{2}, a}(z) \mathcal{O}_{\Delta, J}^{b}(w, \bar{w}) & \sim-\frac{f^{a b c}}{z-w}((2 \bar{h}-1)+\bar{w} \bar{\partial}) \mathcal{O}_{\Delta-1, J}^{c}(w, \bar{w}), \\
\mathcal{K}_{-\frac{1}{2}}^{\frac{3}{2}, a}(z) \mathcal{O}_{\Delta, J}^{b}(w, \bar{w}) & \sim \frac{f^{a b c}}{z-w} \bar{\partial} \mathcal{O}_{\Delta-1, J}^{c}(w, \bar{w}) .
\end{aligned}
$$

The structure here are again identical to that in $[14,16,17,30]$.

As in the graviton case, we can now propose the following Ward identities for soft gluon currents

$$
\begin{aligned}
& \left\langle K^{l, a}(z, \bar{z}) \mathcal{O}_{\Delta_{1}, J_{1}}^{b_{1}}\left(z_{1}, \bar{z}_{1}\right) \cdots \mathcal{O}_{\Delta_{m}, J_{m}}^{b_{m}}\left(z_{m}, \bar{z}_{m}\right)\right\rangle \\
& =\sum_{k=1}^{m} f^{a b_{k} c_{k}} \sum_{s=0}^{1-l} \frac{\left(\bar{z}-\bar{z}_{k}\right)^{s}}{z-z_{k}} \frac{(-1)^{-s+1-l}}{s !(-s+1-l) !} \frac{\Gamma\left(2 \bar{h}_{k}\right)}{\Gamma\left(2 \bar{h}_{k}+l+s-1\right)} \\
& \quad \times \bar{\partial}_{k}^{s}\left\langle\mathcal{O}_{\Delta_{1}, J_{1}}^{b_{1}}\left(z_{1}, \bar{z}_{1}\right) \cdots \mathcal{O}_{\Delta_{k}+l-1, J_{k}}^{c_{k}}\left(z_{k}, \bar{z}_{k}\right) \cdots \mathcal{O}_{\Delta_{m}, J_{m}}^{b_{m}}\left(z_{1}, \bar{z}_{m}\right)\right\rangle,
\end{aligned}
$$

and the chiral Ward identities for chiral gluon currents

$$
\begin{aligned}
& \left\langle\mathcal{K}_{n}^{i, a}(z) \mathcal{O}_{\Delta_{1}, J_{1}}^{b_{1}}\left(z_{1}, \bar{z}_{1}\right) \cdots \mathcal{O}_{\Delta_{m}, J_{m}}^{b_{m}}\left(z_{m}, \bar{z}_{m}\right)\right\rangle \\
& =\sum_{k=1}^{m} \frac{f^{a b_{k} c_{k}}}{z-z_{k}} \sum_{r=0}^{i-1+n}(-1)^{i-1+n}\left(\begin{array}{c}
i-1+n \\
r
\end{array}\right) \frac{\Gamma\left(2 \bar{h}_{k}\right)}{\Gamma\left(2 \bar{h}_{k}+1-i-n+r\right)} \\
& \quad \times \bar{z}_{k}^{r} \bar{\partial}_{k}^{r+i-1-n}\left\langle\mathcal{O}_{\Delta_{1}, J_{1}}^{b_{1}}\left(z_{1}, \bar{z}_{1}\right) \cdots \mathcal{O}_{\Delta_{k}+l-1, J_{k}}^{c_{k}}\left(z_{k}, \bar{z}_{k}\right) \cdots \mathcal{O}_{\Delta_{m}, J_{m}}^{b_{m}}\left(z_{1}, \bar{z}_{m}\right)\right\rangle,
\end{aligned}
$$

where the hard operators in the vector multiplet have helicity $J_{k}= \pm \frac{1}{2}, \pm 1{ }^{15}$ The arguments for the validity of these Ward identities are similar to that in the graviton case we discussed before. And it is easy to check that they are in perfect agreement with the Ward identities for leading and sub-leading soft gluon theorems [20, 30, 31]:

$$
\begin{aligned}
& \left\langle K^{1, a}(z, \bar{z}) \mathcal{O}_{\Delta_{1}, J_{1}}^{b_{1}}\left(z_{1}, \bar{z}_{1}\right) \cdots \mathcal{O}_{\Delta_{m}, J_{m}}^{b_{m}}\left(z_{m}, \bar{z}_{m}\right)\right\rangle \\
& =\sum_{k=1}^{m} \frac{f^{a b_{k} c_{k}}}{z-z_{k}}\left\langle\mathcal{O}_{\Delta_{1}, J_{1}}^{b_{1}}\left(z_{1}, \bar{z}_{1}\right) \cdots \mathcal{O}_{\Delta_{k}, J_{k}}^{c_{k}}\left(z_{k}, \bar{z}_{k}\right) \cdots \mathcal{O}_{\Delta_{m}, J_{m}}^{b_{m}}\left(z_{m}, \bar{z}_{m}\right)\right\rangle,
\end{aligned}
$$

and

$$
\begin{aligned}
& \left\langle K^{0, a}(z, \bar{z}) \mathcal{O}_{\Delta_{1}, J_{1}}^{b_{1}}\left(z_{1}, \bar{z}_{1}\right) \cdots \mathcal{O}_{\Delta_{m}, J_{m}}^{b_{m}}\left(z_{m}, \bar{z}_{m}\right)\right\rangle \\
& =\sum_{k=1}^{m} \frac{f^{a b_{k} c_{k}}}{z-z_{k}}\left[-\left(2 \bar{h}_{k}-1\right)+\left(\bar{z}-\bar{z}_{k}\right) \bar{\partial}_{k}\right]\left\langle\mathcal{O}_{\Delta_{1}, J_{1}}^{b_{1}}\left(z_{1}, \bar{z}_{1}\right) \cdots \mathcal{O}_{\Delta_{k}-1, J_{k}}^{c_{k}}\left(z_{k}, \bar{z}_{k}\right) \cdots \mathcal{O}_{\Delta_{m}, J_{m}}^{b_{m}}\left(z_{m}, \bar{z}_{m}\right)\right\rangle .
\end{aligned}
$$

\subsection{Photon Ward identity}

For Maxwell-matter coupled system, like QED, the scattering amplitudes also factorize when the photon is taken soft. This gives rise to soft photon theorems which are universal at leading and sub-leading orders. We review it in appendix D.

\footnotetext{
${ }^{15}$ More generally, a similar type of Ward identity is supposed to hold even for hard operators belonging to the chiral multiplets which have helicities $J=0, \pm 1 / 2$ and transform in some representation $\mathbf{R}$ of the gauge group, but one needs to replace $f^{a b c}$ with some representation matrix $\left(T_{\mathbf{R}}^{a}\right)_{I J}$.
} 
Now we want to derive the Ward identities associated to soft photons. We start with the following OPE between photon and matter fields which are minimally coupled:

$$
O_{\Delta,+1}\left(z_{1}, \bar{z}_{1}\right) \mathcal{O}_{\Delta^{\prime}, J^{\prime}}\left(z_{2}, \bar{z}_{2}\right) \sim \frac{e}{z_{12}} B\left(\Delta-1, \Delta^{\prime}-J\right) O_{\Delta+\Delta^{\prime}-1, J^{\prime}}\left(z_{2}, \bar{z}_{2}\right) .
$$

This is very similar to the gluon case in (3.1) except that now we need to strip off the color index and use $O_{\Delta,+1}$. Also $O_{\Delta^{\prime}, J^{\prime}}$ is a matter operator with electric charge $e$ under U(1) Maxwell field.

Repeating the same procedure, we obtain the Ward identities

$$
\begin{aligned}
& \left\langle K^{l}(z, \bar{z}) \mathcal{O}_{\Delta_{1}, J_{1}}\left(z_{1}, \bar{z}_{1}\right) \cdots \mathcal{O}_{\Delta_{m}, J_{m}}\left(z_{m}, \bar{z}_{m}\right)\right\rangle \\
& =\sum_{k=1}^{m} e_{k} \sum_{s=0}^{1-l} \frac{\left(\bar{z}-\bar{z}_{k}\right)^{s}}{z-z_{k}} \frac{(-1)^{-s+1-l}}{s !(-s+1-l) !} \frac{\Gamma\left(2 \bar{h}_{k}\right)}{\Gamma\left(2 \bar{h}_{k}+l+s-1\right)} \\
& \quad \times \bar{\partial}_{k}^{s}\left\langle\mathcal{O}_{\Delta_{1}, J_{1}}\left(z_{1}, \bar{z}_{1}\right) \cdots \mathcal{O}_{\Delta_{k}+l-1, J_{k}}\left(z_{k}, \bar{z}_{k}\right) \cdots \mathcal{O}_{\Delta_{m}, J_{m}}\left(z_{m}, \bar{z}_{m}\right)\right\rangle
\end{aligned}
$$

which has similar structure as in the gluon case (4.33). The difference is that Maxwell theory itself is free and we need extra matter fields to interact.

For $l=1,0$, these Ward identities exactly coincide with the leading and sub-leading soft photon theorem in (D.8) after performing Mellin transformation.

We can also obtain the chiral Ward identities. For leading soft photon, we find

$$
\begin{aligned}
& \left\langle\mathcal{K}_{0}^{1}(z) \mathcal{O}_{\Delta_{1}, J_{1}}\left(z_{1}, \bar{z}_{1}\right) \cdots \mathcal{O}_{\Delta_{m}, J_{m}}\left(z_{m}, \bar{z}_{m}\right)\right\rangle \\
& =\sum_{k=1}^{m} \frac{e_{k}}{z-z_{k}}\left\langle\mathcal{O}_{\Delta_{1}, J_{1}}\left(z_{1}, \bar{z}_{1}\right) \cdots O_{\Delta_{k}-1, J_{k}}\left(z_{k}, \bar{z}_{k}\right) \cdots \mathcal{O}_{\Delta_{m}, J_{m}}\left(z_{1}, \bar{z}_{m}\right)\right\rangle
\end{aligned}
$$

which was also previously obtained in [32] by different means.

For sub-leading soft photon, we have two chiral Ward identities

$$
\begin{aligned}
& \left\langle\mathcal{K}_{\frac{1}{2}}^{\frac{3}{2}}(z) \mathcal{O}_{\Delta_{1}, J_{1}}\left(z_{1}, \bar{z}_{1}\right) \cdots \mathcal{O}_{\Delta_{m}, J_{m}}\left(z_{m}, \bar{z}_{m}\right)\right\rangle \\
& =\sum_{k=1}^{m} \frac{-e_{k}}{z-z_{k}}\left[\left(2 \bar{h}_{k}-1\right)+\bar{z}_{k} \bar{\partial}_{k}\right]\left\langle\mathcal{O}_{\Delta_{1}, J_{1}}\left(z_{1}, \bar{z}_{1}\right) \cdots O_{\Delta_{k}-1, J_{k}}\left(z_{k}, \bar{z}_{k}\right) \cdots \mathcal{O}_{\Delta_{m}, J_{m}}\left(z_{m}, \bar{z}_{m}\right)\right\rangle
\end{aligned}
$$

and

$$
\begin{aligned}
& \left\langle\mathcal{K}_{-\frac{1}{2}}^{\frac{3}{2}}(z) \mathcal{O}_{\Delta_{1}, J_{1}}\left(z_{1}, \bar{z}_{1}\right) \cdots \mathcal{O}_{\Delta_{m}, J_{m}}\left(z_{m}, \bar{z}_{m}\right)\right\rangle \\
& =\sum_{k=1}^{m} \frac{e_{k}}{z-z_{k}} \bar{\partial}_{k}\left\langle\mathcal{O}_{\Delta_{1}, J_{1}}\left(z_{1}, \bar{z}_{1}\right) \cdots O_{\Delta_{k}-1, J_{k}}\left(z_{k}, \bar{z}_{k}\right) \cdots \mathcal{O}_{\Delta_{m}, J_{m}}\left(z_{m}, \bar{z}_{m}\right)\right\rangle .
\end{aligned}
$$

In (4.41), if we take a further derivative with respect to $z$ and thus consider the Ward identity associated with $\partial \mathcal{K}_{-\frac{1}{2}}^{\frac{3}{2}}$, then we rediscover the Ward identity found in [33], which was shown to arise from the sub-leading soft photon theorem. 
Magnetic corrections. In the above discussions, we considered the matter particles which are electrically charged under U(1) Maxwell field. It is known that there are magnetic corrections to the soft photon theorem as we review in appendix D. Such corrections have also be understood from the perspective of asymptotic symmetry [34]. It is then natural to ask can we also include the magnetic corrections in our formalism? This turns out to be very easy: we just need to complexify the couplings by replacing $e_{k} \rightarrow e_{k}+i g_{k}$ where $e_{k}, g_{k}$ are the electric and magnetic charges of particles. ${ }^{16}$ Indeed, in the threepoint amplitude between photon and charged matter particle, nothing prevents us from considering a complexified coupling for this three-point amplitude. A complexified coupling just means that the charged particle is a dyon with both electric and magnetic charge. This can be easily understood from electromagnetic duality ${ }^{17}$ which rotates the phase of the wave function of photon with definite helicity; as a result, the three-point coupling also acquires a phase. This simple modification does not affect our derivation above at all except that we need to consider complexified coupling $e_{k} \rightarrow e_{k}+i g_{k}$ in OPE (4.37) and (4.38). The resulting Ward identities with magnetic corrections are thus similarly derived purely from 2D CCFT and are equivalent to the soft photon theorems.

\section{Shadow Ward identities}

In the last section, we derived the infinitely many Ward identities associated to the infinite dimensional soft currents. In this section, we would like to use shadow transformation to derive the infinitely many shadow Ward identities.

Generally, the shadow transformation of operator $O$ with weights $(h, \bar{h})$ is defined as $[35]^{18}$

$$
\widetilde{O}(w, \bar{w}) \equiv \mathbf{S}[O](w, \bar{w})=\int d^{2} z(z-w)^{2 h-2}(\bar{z}-\bar{w})^{2 \bar{h}-2} O(z, \bar{z}) .
$$

As such, the shadow operator $\widetilde{O}$ has holomorphic and anti-holomorphic weights $(1-h, 1-\bar{h})$, or equivalently conformal dimension $2-\Delta$ and spin $-J$.

Compared to Ward identities themselves, the shadow Ward identities play an equally important role in celestial holography. For example, by performing the shadow transformation on the subleading soft graviton current, one gets the stress tensor in celestial CFT $[6,36]$; and the shadow Ward identity at subleading order just coincides with the standard stress tensor Ward identity. We will generalize this construction and derive the shadow Ward identity associated with all the soft currents for graviton, gluon and photon. Furthermore, a general formula of shadow Ward identities will be derived in (6.5), which is also applicable to soft gravitino and soft gluino.

\footnotetext{
${ }^{16}$ The replacing $e_{k} \rightarrow e_{k}+i g_{k}$ is for positive helicity photon; for negative helicity photon, we should replace $e_{k} \rightarrow e_{k}-i g_{k}$.

${ }^{17}$ See [32] for some discussions about electromagnetic duality in this context.

${ }^{18}$ In this section and appendix $\mathrm{B}$, for computational convenience we will treat $z, \bar{z}$ as complex conjugate of each other.
} 


\subsection{Shadow graviton Ward identity}

The graviton Ward identities are derived in (4.17). Let us write down again:

$$
\begin{aligned}
& \left\langle H^{l}(z, \bar{z}) \mathcal{O}_{\Delta_{1}, J_{1}}\left(z_{1}, \bar{z}_{1}\right) \cdots \mathcal{O}_{\Delta_{m}, J_{m}}\left(z_{m}, \bar{z}_{m}\right)\right\rangle \\
& =\sum_{k=1}^{m} \sum_{s=0}^{1-l} \frac{\left(\bar{z}-\bar{z}_{k}\right)^{s+1}}{z-z_{k}} \frac{(-1)^{-s-l}}{s !(-s+1-l) !} \frac{\Gamma\left(2 \bar{h}_{k}+1\right)}{\Gamma\left(2 \bar{h}_{k}+l+s\right)} \\
& \quad \times \bar{\partial}_{k}^{s}\left\langle\mathcal{O}_{\Delta_{1}, J_{1}}\left(z_{1}, \bar{z}_{1}\right) \cdots \mathcal{O}_{\Delta_{k}+l, J_{k}}\left(z_{2}, \bar{z}_{2}\right) \cdots \mathcal{O}_{\Delta_{m}, J_{m}}\left(z_{m}, \bar{z}_{m}\right)\right\rangle .
\end{aligned}
$$

Note that soft graviton current $H^{l}$ has weight $(h, \bar{h})=\left(\frac{l+2}{2}, \frac{l-2}{2}\right)$. Its shadow current is given by (5.1):

$$
\widetilde{H^{l}}(w, \bar{w})=\int d^{2} z(z-w)^{l}(\bar{z}-\bar{w})^{l-4} H^{l}(z, \bar{z}) .
$$

To derive the shadow Ward identities, we need to perform a similar integral on the right hand side of original Ward identities (5.2).

The integral has been computed in appendix B. In particular, specializing (B.17) to the present case, we have

$$
\int d^{2} z(z-w)^{l}(\bar{z}-\bar{w})^{l-4} \frac{\left(\bar{z}-\bar{z}^{\prime}\right)^{s+1}}{z-z^{\prime}}=\frac{(-1)^{s} \pi(s+1) !}{(l-3)(l-2) \cdots(l+s-2)}\left(z^{\prime}-w\right)^{l}\left(\bar{z}^{\prime}-\bar{w}\right)^{l+s-2} .
$$

Plugging this integral into (5.2) and doing some algebra yields the following shadow Ward identities: ${ }^{19}$

$$
\begin{aligned}
& \left\langle\widetilde{H^{l}}(w, \bar{w}) \mathcal{O}_{\Delta_{1}, J_{1}}\left(z_{1}, \bar{z}_{1}\right) \cdots \mathcal{O}_{\Delta_{m}, J_{m}}\left(z_{m}, \bar{z}_{m}\right)\right\rangle \\
& =\frac{(-1)^{l} \pi}{(3-l) !} \sum_{k=1}^{m} \sum_{s=0}^{1-l} \frac{(s+1) \Gamma\left(2 \bar{h}_{k}+1\right)}{\Gamma\left(2 \bar{h}_{k}+l+s\right)} \times\left(w-z_{k}\right)^{l}\left(\bar{w}-\bar{z}_{k}\right)^{l+s-2} \\
& \quad \times \bar{\partial}_{k}^{s}\left\langle\mathcal{O}_{\Delta_{1}, J_{1}}\left(z_{1}, \bar{z}_{1}\right) \cdots \mathcal{O}_{\Delta_{k}+l, J_{k}}\left(z_{k}, \bar{z}_{k}\right) \cdots \mathcal{O}_{\Delta_{m}, J_{m}}\left(z_{m}, \bar{z}_{m}\right)\right\rangle .
\end{aligned}
$$

Let us write down the identities at several leading orders explicitly.

For leading soft graviton $l=1$, we have

$$
\begin{aligned}
& \left\langle\widetilde{H^{1}}(w, \bar{w}) \mathcal{O}_{\Delta_{1}, J_{1}}\left(z_{1}, \bar{z}_{1}\right) \cdots \mathcal{O}_{\Delta_{m}, J_{m}}\left(z_{m}, \bar{z}_{m}\right)\right\rangle \\
& =-\frac{\pi}{2} \sum_{k=1}^{m} \frac{w-z_{k}}{\bar{w}-\bar{z}_{k}}\left\langle\mathcal{O}_{\Delta_{1}, J_{1}}\left(z_{1}, \bar{z}_{1}\right) \cdots \mathcal{O}_{\Delta_{k}+1, J_{k}}\left(z_{k}, \bar{z}_{k}\right) \cdots \mathcal{O}_{\Delta_{m}, J_{m}}\left(z_{m}, \bar{z}_{m}\right)\right\rangle .
\end{aligned}
$$

This coincides with the Ward identity associated with the leading soft (negative helicity) graviton theorem.

For sub-leading soft graviton $l=0$, we have

$$
\begin{aligned}
& \left\langle\frac{3}{\pi} \widetilde{H^{0}}(\bar{w}) \mathcal{O}_{\Delta_{1}, J_{1}}\left(z_{1}, \bar{z}_{1}\right) \cdots \mathcal{O}_{\Delta_{m}, J_{m}}\left(z_{m}, \bar{z}_{m}\right)\right\rangle \\
& =\sum_{k=1}^{m}\left[\frac{\bar{h}_{k}}{\left(\bar{w}-\bar{z}_{k}\right)^{2}}+\frac{\bar{\partial}_{k}}{\bar{w}-\bar{z}_{k}}\right]\left\langle\mathcal{O}_{\Delta_{1}, J_{1}}\left(z_{1}, \bar{z}_{1}\right) \cdots \mathcal{O}_{\Delta_{m}, J_{m}}\left(z_{m}, \bar{z}_{m}\right)\right\rangle .
\end{aligned}
$$

\footnotetext{
${ }^{19}$ It is interesting to note that the holomorphic dependence on shadow current is simply $\left(w-z_{k}\right)^{l}$. So for $l \leq-1$, we may integrate $w$ and reduce $\left(w-z_{k}\right)^{l}$ to $1 /\left(w-z_{k}\right)$. This simplifies the equation a little bit but the physical meaning is not clear.
} 
This becomes the standard Ward identity of anti-holomorphic stress tensor in CFT once we identify $\bar{T}(\bar{w})=\frac{3}{\pi} \widetilde{H^{0}}(\bar{w})$. This stress tensor Ward identity was previously discussed in $[6,36,37] .{ }^{20}$

For sub-sub-leading soft graviton $l=-1$, we find

$$
\begin{aligned}
& \left\langle\widetilde{H^{-1}}(w, \bar{w}) \mathcal{O}_{\Delta_{1}, J_{1}}\left(z_{1}, \bar{z}_{1}\right) \cdots \mathcal{O}_{\Delta_{m}, J_{m}}\left(z_{m}, \bar{z}_{m}\right)\right\rangle \\
& =-\frac{\pi}{4 !} \sum_{k=1}^{m} \frac{1}{w-z_{k}}\left[\frac{2 \bar{h}_{k}\left(2 \bar{h}_{k}-1\right)}{\left(\bar{w}-\bar{z}_{k}\right)^{3}}+\frac{4 \bar{h}_{k} \bar{\partial}_{k}}{\left(\bar{w}-\bar{z}_{k}\right)^{2}}+\frac{3 \bar{\partial}_{k}^{2}}{\bar{w}-\bar{z}_{k}}\right] \\
& \quad \times\left\langle\mathcal{O}_{\Delta_{1}, J_{1}}\left(z_{1}, \bar{z}_{1}\right) \cdots \mathcal{O}_{\Delta_{k}-1, J_{k}}\left(z_{k}, \bar{z}_{k}\right) \cdots \mathcal{O}_{\Delta_{m}, J_{m}}\left(z_{m}, \bar{z}_{m}\right)\right\rangle .
\end{aligned}
$$

\subsection{Shadow photon and gluon Ward identity}

Now we switch to shadow Ward identities for soft photons. The soft photon Ward identities are given in (4.38):

$$
\begin{aligned}
& \left\langle K^{l}(z, \bar{z}) \mathcal{O}_{\Delta_{1}, J_{1}}\left(z_{1}, \bar{z}_{1}\right) \cdots \mathcal{O}_{\Delta_{m}, J_{m}}\left(z_{m}, \bar{z}_{m}\right)\right\rangle \\
& =\sum_{k=1}^{m} e_{k} \sum_{s=0}^{1-l} \frac{\left(\bar{z}-\bar{z}_{k}\right)^{s}}{z-z_{k}} \frac{(-1)^{-s+1-l}}{s !(-s+1-l) !} \frac{\Gamma\left(2 \bar{h}_{k}\right)}{\Gamma\left(2 \bar{h}_{k}+l+s-1\right)} \\
& \quad \times \bar{\partial}_{k}^{s}\left\langle\mathcal{O}_{\Delta_{1}, J_{1}}\left(z_{1}, \bar{z}_{1}\right) \cdots \mathcal{O}_{\Delta_{k}+l-1, J_{k}}\left(z_{k}, \bar{z}_{k}\right) \cdots \mathcal{O}_{\Delta_{m}, J_{m}}\left(z_{m}, \bar{z}_{m}\right)\right\rangle .
\end{aligned}
$$

The soft photon current $K^{l}$ has weight $(h, \bar{h})=\left(\frac{l+1}{2}, \frac{l-1}{2}\right)$. Thus the soft photon shadow current is given by (5.1):

$$
\widetilde{K^{l}}(w, \bar{w})=\int d^{2} z(z-w)^{l-1}(\bar{z}-\bar{w})^{l-3} K^{l}(z, \bar{z}) .
$$

To proceed, we need to use the following integral which has been derived in (B.17):

$$
\int d^{2} z(z-w)^{l-1}(\bar{z}-\bar{w})^{l-3} \frac{\left(\bar{z}-\bar{z}^{\prime}\right)^{s}}{z-z^{\prime}}=\frac{(-1)^{s}(-\pi) s !}{(l-2)(l-2) \cdots(l+s-2)}\left(z^{\prime}-w\right)^{l-1}\left(\bar{z}^{\prime}-\bar{w}\right)^{l+s-2} \text {. }
$$

Plugging this integral into (5.9) gives the following shadow Ward identities:

$$
\begin{aligned}
& \left\langle\widetilde{K^{l}}(w, \bar{w}) \mathcal{O}_{\Delta_{1}, J_{1}}\left(z_{1}, \bar{z}_{1}\right) \cdots \mathcal{O}_{\Delta_{m}, J_{m}}\left(z_{m}, \bar{z}_{m}\right)\right\rangle \\
& =\frac{(-1)^{-l} \pi}{(2-l) !} \sum_{k=1}^{m} e_{k} \sum_{s=0}^{1-l} \frac{\Gamma\left(2 \bar{h}_{k}\right)}{\Gamma\left(2 \bar{h}_{k}+l+s-1\right)}\left(w-z_{k}\right)^{l-1}\left(\bar{w}-\bar{z}_{k}\right)^{l+s-2} \\
& \quad \times \bar{\partial}^{s}\left\langle\mathcal{O}_{\Delta_{1}, J_{1}}\left(z_{1}, \bar{z}_{1}\right) \cdots \mathcal{O}_{\Delta_{k}+l-1, J_{k}}\left(z_{k}, \bar{z}_{k}\right) \cdots \mathcal{O}_{\Delta_{m}, J_{m}}\left(z_{m}, \bar{z}_{m}\right)\right\rangle .
\end{aligned}
$$

More explicitly for leading soft photon $l=1$, we have

$$
\begin{aligned}
& \left\langle\widetilde{K^{1}}(w, \bar{w}) \mathcal{O}_{\Delta_{1}, J_{1}}\left(z_{1}, \bar{z}_{1}\right) \cdots \mathcal{O}_{\Delta_{m}, J_{m}}\left(z_{m}, \bar{z}_{m}\right)\right\rangle \\
& =-\pi \sum_{k=1}^{m} \frac{e_{k}}{\bar{w}-\bar{z}_{k}}\left\langle\mathcal{O}_{\Delta_{1}, J_{1}}\left(z_{1}, \bar{z}_{1}\right) \cdots \mathcal{O}_{\Delta_{m}, J_{m}}\left(z_{m}, \bar{z}_{m}\right)\right\rangle,
\end{aligned}
$$

which is the same as the Ward identity associated with the leading soft photon with negative helicity.

\footnotetext{
${ }^{20}$ Note that the coefficient $3 / \pi$ also matches with previous literature, see e.g. eq. 3.1 of [37] and eq. 4.28 of $[10]$.
} 
While for sub-leading soft photon $l=0$, we have

$$
\begin{aligned}
& \left\langle\widetilde{K^{0}}(w, \bar{w}) \mathcal{O}_{\Delta_{1}, J_{1}}\left(z_{1}, \bar{z}_{1}\right) \cdots \mathcal{O}_{\Delta_{m}, J_{m}}\left(z_{m}, \bar{z}_{m}\right)\right\rangle \\
& =\frac{\pi}{2} \sum_{k=1}^{m} \frac{e_{k}}{w-z_{k}}\left[\frac{2 \bar{h}_{k}-1}{\left(\bar{w}-\bar{z}_{k}\right)^{2}}+\frac{\bar{\partial}_{k}}{\bar{w}-\bar{z}_{k}}\right]\left\langle\mathcal{O}_{\Delta_{1}, J_{1}}\left(z_{1}, \bar{z}_{1}\right) \cdots \mathcal{O}_{\Delta_{k}-1, J_{k}}\left(z_{k}, \bar{z}_{k}\right) \cdots \mathcal{O}_{\Delta_{m}, J_{m}}\left(z_{m}, \bar{z}_{m}\right)\right\rangle .
\end{aligned}
$$

For gluon, their corresponding shadow Ward identities are similarly given by:

$$
\begin{aligned}
& \left\langle\widetilde{K^{l, a}}(w, \bar{w}) \mathcal{O}_{\Delta_{1}, J_{1}}^{b_{1}}\left(z_{1}, \bar{z}_{1}\right) \cdots \mathcal{O}_{\Delta_{m}, J_{m}}^{b_{m}}\left(z_{m}, \bar{z}_{m}\right)\right\rangle \\
& =\frac{(-1)^{-l} \pi}{(2-l) !} \sum_{k=1}^{m} f^{a b_{k} c_{k}} \sum_{s=0}^{1-l} \frac{\Gamma\left(2 \bar{h}_{k}\right)}{\Gamma\left(2 \bar{h}_{k}+l+s-1\right)}\left(w-z_{k}\right)^{l-1}\left(\bar{w}-\bar{z}_{k}\right)^{l+s-2} \\
& \quad \times \bar{\partial}^{s}\left\langle\mathcal{O}_{\Delta_{1}, J_{1}}^{b_{1}}\left(z_{1}, \bar{z}_{1}\right) \cdots \mathcal{O}_{\Delta_{k}+l-1, J_{k}}^{c_{k}}\left(z_{k}, \bar{z}_{k}\right) \cdots \mathcal{O}_{\Delta_{m}, J_{m}}^{b_{m}}\left(z_{m}, \bar{z}_{m}\right)\right\rangle .
\end{aligned}
$$

\section{$6 \quad$ EFT corrections}

In the previous sections, we proposed a method for deriving Ward identities from celestial OPE. With this procedure, we discussed the Ward identities associated with the soft symmetry currents in supersymmetric EYM theory. Although we were considering this specific theory, the Ward identities are supposed to hold more generally as they capture the universal feature of quantum fields. However, they are not always universal; there are various types of correction to the soft theorems due to quantum loops or higher derivative interactions. More specifically, it has been shown in [18] that there are cubic vertices which can modify the sub-sub-leading soft graviton theorem and sub-leading soft photon theorem. These claims are derived in [18] by considering local unitary effective field theory and analyzing all possible local operators. Therefore, the leading and sub-leading soft graviton theorems, as well as the leading soft photon theorem are indeed universal at tree level in EFT as guaranteed by locality and unitarity. ${ }^{21}$

We will reformulate their results in the language of celestial holography. More specifically, we will consider the EFT corrections to our previous Ward identities. The procedure is the same as that in the previous sections. We will first derive a general celestial OPE (6.1) arising from the cubic interaction of three massless spinning particles. Based on this general OPE, we establish the general Ward identities (6.5) and its shadow cousin (6.6). Applying the general results to EFT, we find that the corrections to Ward identities indeed start to appear at sub-sub-leading order for soft graviton and sub-leading order for soft photon.

\subsection{General celestial OPE and Ward identity}

As we derived in appendix $\mathrm{C}$, the leading tree level celestial OPEs arising from cubic vertices of three spinning massless particles take the following general form:

$$
\begin{aligned}
& \mathcal{O}_{\Delta_{1}, J_{1}}\left(z_{1}, \bar{z}_{1}\right) \mathcal{O}_{\Delta_{2}, J_{2}}\left(z_{2}, \bar{z}_{2}\right) \\
& \sim \kappa_{J_{1} J_{2} J_{3}} \frac{\bar{z}_{12}^{J_{1}+J_{2}+J_{3}-1}}{z_{12}} B\left(\Delta_{1}+J_{2}+J_{3}-1, \Delta_{2}+J_{1}+J_{3}-1\right) \mathcal{O}_{\Delta_{3},-J_{3}}\left(z_{2}, \bar{z}_{2}\right),
\end{aligned}
$$

\footnotetext{
${ }^{21}$ The Weinberg's leading soft graviton theorem is even robust against quantum loops, while the rest of soft theorems may suffer from quantum corrections.
} 
where $\Delta_{3}=\Delta_{1}+\Delta_{2}+J_{1}+J_{2}+J_{3}-2$ and $\kappa_{J_{1} J_{2} J_{3}}$ is the coupling constant of the cubic vertex. This is derived in condition $\mathcal{J} \equiv J_{1}+J_{2}+J_{3} \geq 0 .{ }^{22}$ One can check that this OPE agrees with all known celestial OPEs, including those in (2.1)-(2.10).

As before, we also want to sum over all the $\overline{\mathrm{SL}(2, \mathbb{R})}$ descendants. Using $(2.22)$, we get

$$
\begin{aligned}
& \mathcal{O}_{\Delta_{1}, J_{1}}\left(z_{1}, \bar{z}_{1}\right) \mathcal{O}_{\Delta_{2}, J_{2}}\left(z_{2}, \bar{z}_{2}\right) \\
& \sim \kappa_{J_{1} J_{2} J_{3}} \frac{\bar{z}_{12}^{J_{1}+J_{2}+J_{3}-1}}{z_{12}} \int_{0}^{1} d t \mathcal{O}_{\Delta_{3},-J_{3}}\left(z_{2}, \bar{z}_{2}+t \bar{z}_{12}\right) t^{\Delta_{1}+J_{2}+J_{3}-2}(1-t)^{\Delta_{2}+J_{1}+J_{3}-2} .
\end{aligned}
$$

Doing the integral thus gives the OPE where all the $\overline{\mathrm{SL}(2, \mathbb{R})}$ descendant contributions are included:

$$
\begin{aligned}
& \mathcal{O}_{\Delta_{1}, J_{1}}\left(z_{1}, \bar{z}_{1}\right) \mathcal{O}_{\Delta_{2}, J_{2}}\left(z_{2}, \bar{z}_{2}\right) \\
& \sim \frac{\kappa J_{1} J_{2} J_{3}}{z_{12}} \sum_{s=0}^{\infty} \frac{\left(\bar{z}_{12}\right)^{\mathcal{J}+s-1}}{s !} \bar{\partial}^{s} \mathcal{O}_{\Delta_{3},-J_{3}}\left(z_{2}, \bar{z}_{2}\right) B\left(\Delta_{1}+s+J_{2}+J_{3}-1, \Delta_{2}+J_{1}+J_{3}-1\right) .
\end{aligned}
$$

Setting $\Delta_{1} \rightarrow k$ where $k \in \mathbb{Z}$ for bosonic soft current or $k \in \mathbb{Z}+\frac{1}{2}$ for fermionic soft current, we obtain the OPE between soft currents and hard operators

$$
\begin{aligned}
R^{k, J_{1}}\left(z_{1}, \bar{z}_{1}\right) \mathcal{O}_{\Delta_{2}, J_{2}}\left(z_{2}, \bar{z}_{2}\right) \sim & \frac{\kappa_{J_{1} J_{2} J_{3}}}{z_{12}} \sum_{s=0}^{1-k-J_{2}-J_{3}} \frac{\left(\bar{z}_{12}\right)^{\mathcal{J}+s-1}}{s !} \bar{\partial}^{s} \mathcal{O}_{\Delta_{3},-J_{3}}\left(z_{2}, \bar{z}_{2}\right) \\
& \times \frac{(-1)^{\left(1-k-s-J_{2}-J_{3}\right)}}{\left(1-k-s-J_{2}-J_{3}\right) !} \frac{\Gamma\left(\Delta_{2}+J_{1}+J_{3}-1\right)}{\Gamma\left(\Delta_{2}+J_{1}+J_{2}+2 J_{3}+k+s-2\right)},
\end{aligned}
$$

where we used (2.17). The Ward identity can then be easily established by replacing each pair of soft current and hard operator with their OPEs above.

Explicitly, the general formula of Ward identities is given by

$$
\begin{aligned}
& \left\langle R^{l, J}(z, \bar{z}) \mathcal{O}_{\Delta_{1}, J_{1}}\left(z_{1}, \bar{z}_{1}\right) \cdots \mathcal{O}_{\Delta_{m}, J_{m}}\left(z_{m}, \bar{z}_{m}\right)\right\rangle \\
& =\sum_{k=1}^{m} \kappa_{J J_{k} J_{k}^{\prime}}(-1)^{\nu\left(\nu_{1}+\cdots+\nu_{k-1}\right)} \sum_{s=0}^{1-l-J_{k}-J_{k}^{\prime}} \frac{\left(\bar{z}-\bar{z}_{k}\right)^{J+J_{k}+J_{k}^{\prime}+s-1}}{z-z_{k}} \\
& \quad \times \frac{(-1)^{\left(1-l-s-J_{k}-J_{k}^{\prime}\right)}}{s !\left(1-l-s-J_{k}-J_{k}^{\prime}\right) !} \frac{\Gamma\left(\Delta_{k}+J+J_{k}^{\prime}-1\right)}{\Gamma\left(\Delta_{k}+J+J_{k}+2 J_{k}^{\prime}+l+s-2\right)} \\
& \quad \times \bar{\partial}_{k}^{s}\left\langle\mathcal{O}_{\Delta_{1}, J_{1}}\left(z_{1}, \bar{z}_{1}\right) \cdots \mathcal{O}_{\Delta_{k}+l+J+J_{k}+J_{k}^{\prime}-2,-J_{k}^{\prime}}\left(z_{k}, \bar{z}_{k}\right) \cdots \mathcal{O}_{\Delta_{m}, J_{m}}\left(z_{m}, \bar{z}_{m}\right)\right\rangle,
\end{aligned}
$$

and its corresponding shadow cousin is

$$
\begin{aligned}
& \left\langle\widetilde{R^{l, J}}(w, \bar{w}) \mathcal{O}_{\Delta_{1}, J_{1}}\left(z_{1}, \bar{z}_{1}\right) \cdots \mathcal{O}_{\Delta_{m}, J_{m}}\left(z_{m}, \bar{z}_{m}\right)\right\rangle \\
& =\frac{\pi(-1)^{J+l+1}}{(1+J-l) !} \sum_{k=1}^{m} \kappa_{J J_{k} J_{k}^{\prime}}(-1)^{\nu\left(\nu_{1}+\cdots+\nu_{k-1}\right)} \sum_{s=0}^{1-l-J_{k}-J_{k}^{\prime}}\left(w-z_{k}\right)^{l+J-2}\left(\bar{w}-\bar{z}_{k}\right)^{J_{k}+J_{k}^{\prime}+l+s-2} \\
& \quad \times \frac{\left(J+J_{k}+J_{k}^{\prime}+s-1\right) !}{s !} \frac{\Gamma\left(\Delta_{k}+J+J_{k}^{\prime}-1\right)}{\Gamma\left(\Delta_{k}+J+J_{k}+2 J_{k}^{\prime}+l+s-2\right)} \\
& \quad \times \bar{\partial}_{k}^{s}\left\langle\mathcal{O}_{\Delta_{1}, J_{1}}\left(z_{1}, \bar{z}_{1}\right) \cdots \mathcal{O}_{\Delta_{k}+l+J+J_{k}+J_{k}^{\prime}-2,-J_{k}^{\prime}}\left(z_{k}, \bar{z}_{k}\right) \cdots \mathcal{O}_{\Delta_{m}, J_{m}}\left(z_{m}, \bar{z}_{m}\right)\right\rangle
\end{aligned}
$$

\footnotetext{
${ }^{22}$ In case $\mathcal{J} \leq 0$, a similar OPE can be obtained by flipping the spin $J_{i} \rightarrow-J_{i}$ and exchanging $z_{i} \leftrightarrow \bar{z}_{i}$.
} 
where to take into account the statistics we introduce $\nu_{i}=\left(2 J_{i}\right) \bmod 2=0,1$ for bosonic and fermionic operators, respectively. The chiral Ward identities can also be easily obtained by performing mode expansion using (2.26) and (2.27).

This establishes the general celestial OPEs and Ward identities. Our discussions in the previous sections just correspond to the special case of the above formulae. For soft graviton, we have $J=2, J_{k}=-J_{k}^{\prime}$; for soft gluon/photon, we have $J=1, J_{k}=-J_{k}^{\prime}$. These formulae are also applicable to fermionic soft current. In the case of minimal coupling, we have $J=3 / 2, J_{k}-1 / 2=-J_{k}^{\prime}$ for soft gravitino, and $J=1 / 2, J_{k}-1 / 2=-J_{k}^{\prime}$ for soft gluino/photino. Inserting these values to the above formulae, we get infinitely many Ward identities corresponding to the fermionic symmetries.

In the next two subsections, we will use the general results here to discuss the corrections to Ward identities for sub-leading soft photon and sub-sub-leading soft graviton based on [18].

\subsection{EFT correction to photon Ward identity}

Let us first discuss the case of photon $J_{1}=1$. Following [18], the EFT corrections appear at $J_{2}+J_{3}=1$. The corresponding celestial OPE reads (6.1):

$$
\mathcal{O}_{\Delta_{1},+1}\left(z_{1}, \bar{z}_{1}\right) \mathcal{O}_{\Delta_{2}, J_{2}}\left(z_{2}, \bar{z}_{2}\right) \sim \frac{\bar{z}_{12}}{z_{12}} B\left(\Delta_{1}, \Delta_{2}-J_{2}+1\right) \mathcal{O}_{\Delta_{1}+\Delta_{2}, J_{2}-1}
$$

where we suppress the coupling constant for simplicity. Specializing (6.4) to the present case, we get the OPE between soft photon current and hard operator

$$
K^{k}\left(z_{1}, \bar{z}_{1}\right) \mathcal{O}_{\Delta_{2}, J_{2}}\left(z_{2}, \bar{z}_{2}\right) \sim \frac{\bar{z}_{12}}{z_{12}} \sum_{s=0}^{-k} \frac{\left(\bar{z}_{12}\right)^{s}}{s !} \bar{\partial}^{s} O_{\Delta_{2}+k, J_{2}-1}\left(z_{2}, \bar{z}_{2}\right) \frac{(-1)^{-k-s}}{(-k-s) !} \frac{\Gamma\left(\Delta_{2}-J_{2}+1\right)}{\Gamma\left(\Delta_{2}-J_{2}+k+s+1\right)} .
$$

The resulting Ward identity is

$$
\begin{aligned}
\left\langle K^{l}(z, \bar{z}) \mathcal{O}_{\Delta_{1}, J_{1}}\left(z_{1}, \bar{z}_{1}\right) \cdots \mathcal{O}_{\Delta_{m}, J_{m}}\left(z_{m}, \bar{z}_{m}\right)\right\rangle \\
=\sum_{k=1}^{m} \sum_{s=0}^{-l} \frac{\left(\bar{z}-\bar{z}_{k}\right)^{s+1}}{z-z_{k}} \frac{(-1)^{-l-s}}{s !(-l-s) !} \frac{\Gamma\left(\Delta_{k}-J_{k}+1\right)}{\Gamma\left(\Delta_{k}-J_{k}+l+s+1\right)} \\
\quad \times \bar{\partial}_{k}^{s}\left\langle\mathcal{O}_{\Delta_{1}, J_{1}}\left(z_{1}, \bar{z}_{1}\right) \cdots \mathcal{O}_{\Delta_{k}+l, J_{k}-1}\left(z_{k}, \bar{z}_{k}\right) \cdots \mathcal{O}_{\Delta_{m}, J_{m}}\left(z_{m}, \bar{z}_{m}\right)\right\rangle .
\end{aligned}
$$

Note that in practice we need to combine the Ward identities for all the interactions together.

Let us look at (6.9) in more detail. At leading order $l=1$, we see it has no effect. At sub-leading order $l=0$, it become non-trivial

$$
\begin{aligned}
& \left\langle K^{0}(z, \bar{z}) \mathcal{O}_{\Delta_{1}, J_{1}}\left(z_{1}, \bar{z}_{1}\right) \cdots \mathcal{O}_{\Delta_{m}, J_{m}}\left(z_{m}, \bar{z}_{m}\right)\right\rangle \\
& =\sum_{k=1}^{m} \frac{\bar{z}-\bar{z}_{k}}{z-z_{k}}\left\langle\mathcal{O}_{\Delta_{1}, J_{1}}\left(z_{1}, \bar{z}_{1}\right) \cdots \mathcal{O}_{\Delta_{k}, J_{k}-1}\left(z_{k}, \bar{z}_{k}\right) \cdots \mathcal{O}_{\Delta_{m}, J_{m}}\left(z_{m}, \bar{z}_{m}\right)\right\rangle,
\end{aligned}
$$

which indeed agree with corrections to soft theorem found in [18] after a Mellin transformation. 
In terms of chiral soft photon currents, the corresponding chiral Ward identities are:

$$
\begin{aligned}
& \left\langle\mathcal{K}_{n}^{i}(z) \mathcal{O}_{\Delta_{1}, J_{1}}\left(z_{1}, \bar{z}_{1}\right) \cdots \mathcal{O}_{\Delta_{m}, J_{m}}\left(z_{m}, \bar{z}_{m}\right)\right\rangle \\
& =\sum_{k=1}^{m} \frac{1}{z-z_{k}} \sum_{r=\max (0, n+2-i)}^{n+i-1}\left(\begin{array}{c}
i+n-1 \\
r
\end{array}\right) \frac{(-1)^{i+n-1}(r+i-n-1) \Gamma\left(\Delta_{k}-J_{k}+1\right)}{\Gamma\left(\Delta_{k}-J_{k}+r-i-n+2\right)} \\
& \quad \times \bar{z}_{k}^{r} \bar{\partial}_{k}^{r+i-n-2}\left\langle\mathcal{O}_{\Delta_{1}, J_{1}}\left(z_{1}, \bar{z}_{1}\right) \cdots \mathcal{O}_{\Delta_{k}-2 i+3, J_{k}-1}\left(z_{k}, \bar{z}_{k}\right) \cdots \mathcal{O}_{\Delta_{m}, J_{m}}\left(z_{m}, \bar{z}_{m}\right)\right\rangle .
\end{aligned}
$$

\subsection{EFT correction to graviton Ward identity}

Now we switch to the case of graviton $J_{1}=2$. Following [18], the EFT corrections appear at $J_{2}+J_{3}=2$. The corresponding celestial OPE reads (6.1):

$$
\mathcal{O}_{\Delta_{1},+2}\left(z_{1}, \bar{z}_{1}\right) \mathcal{O}_{\Delta_{2}, J_{2}}\left(z_{2}, \bar{z}_{2}\right) \sim \frac{\bar{z}_{12}^{3}}{z_{12}} B\left(\Delta_{1}+1, \Delta_{2}-J_{2}+3\right) \mathcal{O}_{\Delta_{1}+\Delta_{2}+2, J_{2}-2}\left(z_{2}, \bar{z}_{2}\right),
$$

where the EFT coupling is again suppressed.

Now the soft graviton current has OPE (6.4)

$$
\begin{aligned}
& H^{k}\left(z_{1}, \bar{z}_{1}\right) \mathcal{O}_{\Delta_{2}, J_{2}}\left(z_{2}, \bar{z}_{2}\right) \\
& \sim \frac{\bar{z}_{12}^{3}}{z_{12}} \sum_{s=0}^{-k-1} \frac{\left(\bar{z}_{12}\right)^{s}}{s !} \bar{\partial}^{s} O_{\Delta_{2}+k+2, J_{2}-2}\left(z_{2}, \bar{z}_{2}\right) \frac{(-1)^{-k-s-1}}{(-k-s-1) !} \frac{\Gamma\left(\Delta_{2}-J_{2}+3\right)}{\Gamma\left(\Delta_{2}-J_{2}+k+s+4\right)}
\end{aligned}
$$

The resulting Ward identity is

$$
\begin{aligned}
& \left\langle H^{l}(z, \bar{z}) \mathcal{O}_{\Delta_{1}, J_{1}}\left(z_{1}, \bar{z}_{1}\right) \cdots \mathcal{O}_{\Delta_{m}, J_{m}}\left(z_{m}, \bar{z}_{m}\right)\right\rangle \\
& =\sum_{k=1}^{m} \sum_{s=0}^{-l-1} \frac{\left(\bar{z}-\bar{z}_{k}\right)^{s+3}}{z-z_{k}} \frac{(-1)^{-l-s-1}}{s !(-l-s-1) !} \frac{\Gamma\left(\Delta_{k}-J_{k}+3\right)}{\Gamma\left(\Delta_{k}-J_{k}+l+s+4\right)} \\
& \quad \times \bar{\partial}_{k}^{s}\left\langle\mathcal{O}_{\Delta_{1}, J_{1}}\left(z_{1}, \bar{z}_{1}\right) \cdots \mathcal{O}_{\Delta_{k}+l+2, J_{k}-2}\left(z_{k}, \bar{z}_{k}\right) \cdots \mathcal{O}_{\Delta_{m}, J_{m}}\left(z_{m}, \bar{z}_{m}\right)\right\rangle .
\end{aligned}
$$

At leading and sub-leading order $l=1,0$, it has no effect. At sub-sub-leading order $l=-1$, we find

$$
\begin{aligned}
& \left\langle H^{-1}(z, \bar{z}) \mathcal{O}_{\Delta_{1}, J_{1}}\left(z_{1}, \bar{z}_{1}\right) \cdots \mathcal{O}_{\Delta_{m}, J_{m}}\left(z_{m}, \bar{z}_{m}\right)\right\rangle \\
& =\sum_{k=1}^{m} \frac{\left(\bar{z}-\bar{z}_{k}\right)^{3}}{z-z_{k}}\left\langle\mathcal{O}_{\Delta_{1}, J_{1}}\left(z_{1}, \bar{z}_{1}\right) \cdots \mathcal{O}_{\Delta_{k}+1, J_{k}-2}\left(z_{k}, \bar{z}_{k}\right) \cdots \mathcal{O}_{\Delta_{m}, J_{m}}\left(z_{m}, \bar{z}_{m}\right)\right\rangle .
\end{aligned}
$$

One can show that this is consistent with the corrections to soft graviton theorem found in [18] after doing a Mellin transformation.

As before we can also establish chiral Ward identities:

$$
\begin{aligned}
& \left\langle\mathcal{H}_{n}^{i}(z) \mathcal{O}_{\Delta_{1}, J_{1}}\left(z_{1}, \bar{z}_{1}\right) \cdots \mathcal{O}_{\Delta_{m}, J_{m}}\left(z_{m}, \bar{z}_{m}\right)\right\rangle \\
& =\sum_{k=1}^{m} \frac{1}{z-z_{k}} \sum_{r=\max (0, n+4-i)}^{n+i-1}(-1)^{i+n+1}\left(\begin{array}{c}
i+n-1 \\
r
\end{array}\right) \frac{(r+i-n-1)(r+i-n-2)(r+i-n-3) \Gamma\left(\Delta_{2}-J_{2}+3\right)}{\Gamma\left(\Delta_{2}-J_{2}+r-i-n+4\right)} \\
& \quad \times \bar{z}_{k}^{r} \bar{\partial}_{k}^{r+i-n-4}\left\langle\mathcal{O}_{\Delta_{1}, J_{1}}\left(z_{1}, \bar{z}_{1}\right) \cdots \mathcal{O}_{\Delta_{k}-2 i+6, J_{k}-2}\left(z_{k}, \bar{z}_{k}\right) \cdots \mathcal{O}_{\Delta_{m}, J_{m}}\left(z_{m}, \bar{z}_{m}\right)\right\rangle .
\end{aligned}
$$




\subsection{Absence of EFT correction to holographic chiral algebra}

Finally, we want to understand whether there are corrections to holographic chiral algebra based on the EFT framework and especially the result in [18].

In [18], the authors considered cubic vertices involving three massless particles, and take one of them soft, which we will label as particle 1 . Then they define $\beta=J_{1}-J_{2}-J_{3}+1$. Note the mass dimension of the coupling constant for this vertex is given by $\beta-2 J_{1}=1-\mathcal{J}$. Obviously, we have $\beta, \mathcal{J} \in \mathbb{Z}$ in order to have a bosonic effective operator.

In [18] it was argued that in local unitary EFT, any cubic vertex should satisfy $\beta<4$. What's more, if the vertex involves photons, a stronger condition is $\beta \leq 2$. This immediately implies that no local cubic interactions involving photons, gravitinos, or gravitons are allowed if the sum of their helicities vanishes. This means that in our OPE (6.1), the pole of the form $1 /\left(z_{12} \bar{z}_{12}\right)$ is forbidden in gravitational or Maxwell-matter theory. ${ }^{23}$

When the soft particle 1 is graviton, namely $J_{1}=2$, the condition $\beta<4$ gives $\beta=3,2,1, \cdots$. For $\beta=3$, we have $J_{2}+J_{3}=0$. This just corresponds to the universal gravitational coupling between graviton and matter fields, whose celestial OPE is given in (2.13). For $\beta=2$ and thus $J_{2}+J_{3}=1$, it was argued in [18] that no effective operator satisfying this condition is allowed. For $\beta=1$ and thus $J_{2}+J_{3}=2$, the full list of EFT operators is given by [18]

$$
\phi R_{\mu \nu \rho \sigma} R^{\mu \nu \rho \sigma}, \quad R_{\mu \nu \rho \sigma} F^{\mu \nu} F^{\rho \sigma}, \quad R^{\mu \nu \rho \sigma} \bar{\psi}_{\rho} \gamma_{\mu \nu} \partial_{\sigma} \chi
$$

They can modify the sub-sub-leading soft graviton theorem and the Ward identities as we discussed before.

When the soft particle 1 is photon, namely $J_{1}=1$, the allowed values for $\beta$ are $\beta=2,1, \cdots$. For $\beta=2$ and thus $J_{2}+J_{3}=0$, this is just the minimal coupling between photon and charged matter, whose celestial OPE is described in (4.37). For $\beta=1$ and thus $J_{2}+J_{3}=1$, the full list of allowed effective field theory operators is [18]

$$
\bar{\chi} \gamma^{\mu \nu} F_{\mu \nu} \chi, \quad \phi F_{\mu \nu} F^{\mu \nu}, \quad \phi F_{\mu \nu} \tilde{F}^{\mu \nu}, \quad \bar{\psi}_{\mu} F_{\nu \rho} \gamma^{\mu \nu \rho} \chi, \quad h_{\mu \nu}\left(F^{\mu \rho} F_{\rho}^{\nu}-\frac{1}{4} \eta^{\mu \nu} F_{\rho \sigma} F^{\rho \sigma}\right) .
$$

This type of vertex can modify the sub-leading soft photon theorem and Ward identity as we discussed already.

Now we want to see whether there are corrections to the holographic chiral algebra from the effective field theory operators we listed above. Since our formalism can only deal with positive helicity soft particles, we need to restrict to $J_{1}, J_{2},-J_{3}>0$ in (6.1). It is easy to see that this is possible only for the last two effective operators in (6.18), which have $J_{2}=3 / 2, J_{3}=-1 / 2$ and $J_{2}=2, J_{3}=-1$, respectively. But these are just the gravitational coupling between gravity and photon, which we have considered already. Therefore, all the EFT operators up to this order have no effects on holographic chiral algebra.

One might still worry about even higher derivative operators at smaller $\beta$ which we did not consider above; they may modify the Ward identities at even less leading orders.

\footnotetext{
${ }^{23}$ It would be very interesting to translate the principles of locality, unitarity and causality into some principles in celestial CFT. Then we may use the principles in $2 \mathrm{D}$ to establish these claims.
} 
However, the holographic chiral algebra is generated by the soft graviton currents up to sub-sub-leading order and soft photon currents up to sub-leading order. ${ }^{24}$ Now that there are no EFT corrections at these several leading orders, the higher orders should also not be affected. To conclude, in case we can apply our formalism, any effective operators can not modify the holographic chiral algebra.

\section{Conclusion}

To summarize, in this paper, we studied many aspects of symmetry in celestial holography by deriving the holographic chiral algebra and the associated Ward identities. These symmetries are hidden in the traditional framework of S-matrix, but play important roles in governing the consistency of quantum fields. Inspired by [11], we established a general and systematic framework to reveal these hidden symmetries by making full use of the techniques in conformal field theory. The input of this formalism is the celestial OPEs, while the output is an infinite-dimensional chiral symmetry algebra as well as their corresponding Ward identities. Moreover, we also derived a general formula for tree-level leading order celestial OPE arising from cubic interactions of three spinning massless particles. As a result, we found a general formula of Ward identities (6.5) and (6.6).

In spite of various results in this paper, many interesting questions remain to be further explored. Maybe the most important question is to understand the role of soft particles with negative helicity. Solving this question may finally enable us to discover the full symmetry algebra.

It would be also useful to understand the infinite Ward identities from the traditional momentum space perspective. In [38, 39], an infinite set of soft theorems was found for photon and graviton. And in the MHV sector of gravity, [29] also found infinitely many conformally soft theorems. These soft theorems are likely to be equivalent to our Ward identities, but it remains to check the equivalence explicitly.

Furthermore, the Ward identities we established in this paper have a single current insertion and correspond to the single soft theorems. However, there are also many types of double and multiple soft theorems in gauge theory and gravity. It is natural to ask whether we can also establish the Ward identities with multiple current insertions which are equivalent to the multiple soft theorems.

Another interesting direction is to generalize our framework here to higher dimensions. Although the soft theorems and collinear factorizations of amplitudes are also well studied in higher dimensions, the celestial holography in higher dimensions is largely unexplored. Many results in 4D should be generalizable to higher dimensions. In particular, the equivalence between sub-leading soft graviton theorem and stress tensor Ward identity has been established in [40]. This may be a good starting point for a systematic exploration of symmetry and Ward identity in higher dimensions.

Finally, it is vital to fully understand the corrections to the holographic chiral algebra and Ward identities from quantum loops and higher derivative interactions. We attempted

\footnotetext{
${ }^{24}$ This comes from the fact that celestial OPEs are fully determined by soft theorems up to these orders [17].
} 
to address this question in EFT, but our analysis is restricted to the tree level case with positive soft particles only. We still need to understand the negative soft particles. On the other hand, although the sub-leading soft graviton theorem is not corrected in EFT, it nevertheless suffers quantum correction which has been shown to be one-loop exact [41]. ${ }^{25}$ In [42], this one-loop exact soft theorem was further translated into the loop-corrected stress tensor Ward identity. Since in this paper we have established a systematic framework for Ward identities, it is thus very interesting to incorporate quantum corrections into our formalism and then re-derive the loop-corrected stress tensor Ward identity. This would also allow us to derive the deformation of the holographic chiral algebra. In particular, in the graviton case, the classical $w_{1+\infty}$ algebra is supposed to be deformed to some type of $W_{1+\infty}$ algebra at quantum level.

\section{Acknowledgments}

We would like to thank Ana-Maria Raclariu, Andrew Strominger, and Tomasz Taylor for correspondence. This work was supported by the Royal Society grant, "Relations, Transformations, and Emergence in Quantum Field Theory", and by the Science and Technology Facilities Council (STFC) Consolidated Grant ST/T000686/1 "Amplitudes, strings \& duality".

\section{A Celestial super-OPEs in terms of celestial superfields}

In (2.1)-(2.10), we wrote down explicitly the celestial OPEs for all the component fields in supersymmetric EYM theory. In this appendix we would like to rewrite all the OPEs in a manifest supersymmetric way by introducing the following celestial on-shell superfields [21, 22]:

$$
\begin{aligned}
U_{\Delta}^{a}(z, \bar{z}, \eta) & =\mathcal{O}_{\Delta,+1}^{a}(z, \bar{z})+\eta \mathcal{O}_{\Delta,+\frac{1}{2}}^{a}(z, \bar{z}), \\
W_{\Delta}(z, \bar{z}, \eta) & =\mathcal{O}_{\Delta,+2}(z, \bar{z})+\eta \mathcal{O}_{\Delta,+\frac{3}{2}}(z, \bar{z}),
\end{aligned}
$$

where $\eta$ is the anti-commuting Grassmann variable.

As in the case of $\mathcal{N}=4$ SYM theory [22], it is natural to write down the following super-OPEs:

$$
\begin{aligned}
U_{\Delta_{1}}^{a}\left(z_{1}, \bar{z}_{1}, \eta_{1}\right) U_{\Delta_{2}}^{b}\left(z_{2}, \bar{z}_{2}, \eta_{2}\right) & \sim \frac{f^{a b c}}{z_{12}} U_{\Delta_{1}+\Delta_{2}-1}^{c}\left(z_{2}, \bar{z}_{2}, \eta_{1} e^{\frac{1}{2} \partial_{\Delta_{1}}}+\eta_{2} e^{\frac{1}{2} \partial_{\Delta_{2}}}\right) B\left(\Delta_{1}-1, \Delta_{2}-1\right), \\
W_{\Delta_{1}}\left(z_{1}, \bar{z}_{1}, \eta_{1}\right) W_{\Delta_{2}}\left(z_{2}, \bar{z}_{2}, \eta_{2}\right) & \sim-\frac{\bar{z}_{12}}{z_{12}} W_{\Delta_{1}+\Delta_{2}}\left(z_{2}, \bar{z}_{2}, \eta_{1} e^{\frac{1}{2} \partial_{\Delta_{1}}}+\eta_{2} e^{\frac{1}{2} \partial_{\Delta_{2}}}\right) B\left(\Delta_{1}-1, \Delta_{2}-1\right), \\
U_{\Delta_{1}}^{a}\left(z_{1}, \bar{z}_{1}, \eta_{1}\right) W_{\Delta_{2}}\left(z_{2}, \bar{z}_{2}, \eta_{2}\right) & \sim-\frac{\bar{z}_{12}}{z_{12}} U_{\Delta_{1}+\Delta_{2}}^{a}\left(z_{2}, \bar{z}_{2}, \eta_{1} e^{\frac{1}{2} \partial_{\Delta_{1}}}+\eta_{2} e^{\frac{1}{2} \partial_{\Delta_{2}}}\right) B\left(\Delta_{1}, \Delta_{2}-1\right) .
\end{aligned}
$$

\footnotetext{
${ }^{25}$ More precisely, the IR divergent part is proved to be one-loop exact, while the IR finite part has only been shown to be one-loop exact in some explicit examples. We thank Congkao Wen for discussion on this point.
} 
By expanding the Grassmann parameter, it is easy to verify that the three super-OPEs above agree exactly with the component OPE in (2.1)-(2.10).

The supersymmetry generators acting on these celestial on-shell superfields are given by $[21,22]$ :

$$
Q^{\alpha}=\left(\begin{array}{l}
1 \\
z
\end{array}\right)^{\alpha} e^{\frac{1}{2} \partial_{\Delta}} \eta, \quad \tilde{Q}^{\dot{\alpha}}=\left(\begin{array}{l}
1 \\
\bar{z}
\end{array}\right)^{\dot{\alpha}} e^{\frac{1}{2} \partial_{\Delta}} \partial_{\eta},
$$

which satisfy the standard commutation relation of supersymmetry algebra

$$
\left\{Q^{\alpha}, \tilde{Q}^{\dot{\alpha}}\right\}=P^{\alpha \dot{\alpha}}, \quad P^{\alpha \dot{\alpha}} \equiv P^{\mu} \sigma_{\mu}^{\alpha \dot{\alpha}}=\left(\begin{array}{l}
1 \\
z
\end{array}\right)^{\alpha}\left(\begin{array}{l}
1 \\
\bar{z}
\end{array}\right)^{\dot{\alpha}} e^{\partial_{\Delta}}
$$

where $\alpha, \dot{\alpha}=1,2$. Applying (A.6) to superfields (A.1) and (A.2), we obtain the susy transformation rules of all the component operators, as shown in (2.11) and (2.12).

\section{B Useful integrals}

In this appendix we derive some useful integrals which are crucial for shadow transformation.

\section{B.1 Seed formula}

We first want to compute ${ }^{26}$

$$
I\left(z_{1}, z_{2}\right)=\int d^{2} z \frac{1}{\left(z-z_{1}\right)\left(\bar{z}-\bar{z}_{2}\right)}=\int d^{2} z \frac{\left(\bar{z}-\bar{z}_{1}\right)\left(z-z_{2}\right)}{\left|z-z_{1}\right|^{2}\left|\bar{z}-\bar{z}_{2}\right|^{2}} .
$$

Using Feynman parametrization, ${ }^{27}$ we can rewrite the integral as

$$
\begin{aligned}
I\left(z_{1}, z_{2}\right) & =\int_{0}^{1} d u \int d^{2} z \frac{\left(\bar{z}-\bar{z}_{1}\right)\left(z-z_{2}\right)}{\left(u\left|z-z_{1}\right|^{2}+(1-u)\left|\bar{z}-\bar{z}_{2}\right|^{2}\right)^{2}} \\
& =\int_{0}^{1} d u \int d^{2} w \frac{\left(\bar{w}-(1-u) \bar{z}_{12}\right)\left(w+u z_{12}\right)}{\left(|w|^{2}+u(1-u)\left|z_{12}\right|^{2}\right)^{2}} \\
& =\int_{0}^{1} d u \int d^{2} w \frac{|w|^{2}-u(1-u)\left|\bar{z}_{12}\right|^{2}+u \bar{w} z_{12}-(1-u) w \bar{z}_{12}}{\left(|w|^{2}+u(1-u)\left|z_{12}\right|^{2}\right)^{2}},
\end{aligned}
$$

where we change variable to $w=z+u z_{1}+(1-u) z_{2}$. In the last expression, the last two terms in the numerator is supposed to have no contribution because they are odd function of $w, \bar{w}$. The resulting integral is divergent, so we need to regularize it. We analytically

\footnotetext{
${ }^{26}$ Note that $z, \bar{z}$ are complex conjugate of each other in this appendix.

${ }^{27}$ Namely:

$$
\frac{1}{A B}=\int_{0}^{1} d u \frac{1}{(u A+(1-u) B)^{2}} .
$$
}


continue the dimension from 2 to $d=2+\epsilon$, then the integral becomes

$$
\begin{aligned}
& I\left(z_{1}, z_{2}\right)=\int_{0}^{1} d u \int d \rho \rho^{d-1} V_{d-1} \frac{\rho^{2}-u(1-u)\left|\bar{z}_{12}\right|^{2}}{\left(\rho^{2}+u(1-u)\left|z_{12}\right|^{2}\right)^{2}} \\
&=\frac{2 \pi^{d / 2}}{\Gamma(d / 2)} \int_{0}^{1} d u \frac{(d-1) \pi\left(u(1-u)\left|\bar{z}_{12}\right|^{2}\right)^{\frac{d}{2}-1}}{2 \sin \frac{d \pi}{2}} \quad(\text { for } d<2) \\
&=\frac{2^{2-d} \pi^{\frac{d+3}{2}}\left|z_{12}\right|^{d-1}}{\Gamma\left(\frac{d-1}{2}\right) \sin \frac{d \pi}{2}} \\
& \stackrel{\epsilon \rightarrow 0}{\longrightarrow}-\pi\left(\frac{2}{\epsilon}+\ln \pi+\gamma_{E}+\ln \left|z_{12}\right|^{2}+\mathcal{O}(\epsilon)\right),
\end{aligned}
$$

where $V_{d-1}$ is the area of $d$-1-dimensional sphere $S^{d-1}$. We can regard it as a seed formula and generate many other integrals by taking derivative with respect to $z_{1}, z_{2}$.

\section{B.2 General formula}

The integrals relevant for shadow transformation generally take the following form

$$
I_{s}^{(A, B)}\left(w, z^{\prime}\right)=\int d^{2} z(z-w)^{A}(\bar{z}-\bar{w})^{B} \frac{\left(\bar{z}-\bar{z}^{\prime}\right)^{s}}{z-z^{\prime}} .
$$

Taking derivative with respect to $\bar{z}^{\prime}$, we get ${ }^{28}$

$$
\partial_{\bar{z}^{\prime}} I_{s}^{(A, B)}\left(w, z^{\prime}\right)=-s I_{s-1}^{(A, B)}\left(w, z^{\prime}\right), \quad s>0 .
$$

Using this formula iteratively, we get

$$
\partial_{\bar{z}^{\prime}}^{s} I_{s}^{(A, B)}\left(w, z^{\prime}\right)=(-1)^{s} s ! I_{0}^{(A, B)}\left(w, z^{\prime}\right), \quad s \in \mathbb{N} .
$$

Furthermore, we have

$$
\begin{aligned}
\partial_{\bar{z}^{\prime}} I_{0}^{(A, B)}\left(w, z^{\prime}\right) & =\int d^{2} z(z-w)^{A}(\bar{z}-\bar{w})^{B} \partial_{\bar{z}^{\prime}} \frac{1}{z-z^{\prime}} \\
& =\int d^{2} z(z-w)^{A}(\bar{z}-\bar{w})^{B}(-\pi) \delta^{(2)}\left(z-z^{\prime}\right) \\
& =(-\pi)\left(z^{\prime}-w\right)^{A}\left(\bar{z}^{\prime}-\bar{w}\right)^{B}
\end{aligned}
$$

where we used the formulae

$$
\partial_{\bar{z}} \frac{1}{z}=\pi \delta^{(2)}(z), \quad \delta^{(2)}(z)=\delta(x) \delta(y), \quad d^{2} z=d x d y, \quad z=x+\mathrm{i} y, \quad \int d^{2} z f(z) \delta^{(2)}(z)=f(0) .
$$

Making use of translational invariance and $\mathrm{SL}(2, \mathbb{C})$ invariance, the integral (B.9) is supposed to have the following structure ${ }^{29}$

$$
I_{s}^{(A, B)}\left(w, z^{\prime}\right)=c_{s}^{(A, B)} \times\left(z^{\prime}-w\right)^{A}\left(\bar{z}^{\prime}-\bar{w}\right)^{B+s+1},
$$

\footnotetext{
${ }^{28}$ Note that $\partial_{\bar{z}^{\prime}}$ can hit $1 /\left(z-z^{\prime}\right)$ and generates delta-function $\delta^{(2)}\left(z-z^{\prime}\right)$, but this has vanishing contribution because of $\left(\bar{z}-\bar{z}^{\prime}\right)^{s}$ as long as $s>0$.

${ }^{29}$ This might fail if the exponent is zero. More specifically, if $A=0$, we may have $\log \left(z^{\prime}-w\right)$ instead of $\left(z^{\prime}-w\right)^{0}$. And similarily for anti-holomorphic part $\bar{z}^{\prime}-\bar{w}$. Besides, there may be also some contact-terms in the form $\partial^{\#} \delta\left(z^{\prime}-w\right)$, but this can only contribute in the coincident limit. They have no contribution in our discussion of Ward-identities as long as all the points are distinct, so we ignore this type of contribution.
} 
where $c_{s}^{(A, B)}$ is a constant. We can use relations (B.11) and (B.14) to determine the constant. As a consequence, we find

$$
\begin{aligned}
I_{s}^{(A, B)}\left(w, z^{\prime}\right) & =\int d^{2} z(z-w)^{A}(\bar{z}-\bar{w})^{B} \frac{\left(\bar{z}-\bar{z}^{\prime}\right)^{s}}{z-z^{\prime}} \\
& =\frac{(-1)^{s}(-\pi) s !}{(B+1)(B+2) \cdots(B+s+1)}\left(z^{\prime}-w\right)^{A}\left(\bar{z}^{\prime}-\bar{w}\right)^{B+s+1}
\end{aligned}
$$

\section{B.3 Some examples}

In this subsection we would like to evaluate some integrals explicitly which are relevant for the discussion in the main body. We will use the techniques established in the previous two subsections which are independent. The agreement of different approaches justify our prescription in computing the integral.

We first would like to compute the following integral:

$$
\begin{aligned}
I_{1}^{(0,-4)}\left(w, z^{\prime}\right) & =\int d^{2} z \frac{1}{(\bar{z}-\bar{w})^{4}} \frac{\bar{z}-\bar{z}^{\prime}}{z-z^{\prime}}=\int d^{2} z \frac{1}{(\bar{z}-\bar{w})^{3}\left(z-z^{\prime}\right)}+\left(\bar{w}-\bar{z}^{\prime}\right) \int d^{2} z \frac{1}{(\bar{z}-\bar{w})^{4}\left(z-z^{\prime}\right)} \\
& =\left(\frac{1}{2} \partial_{\bar{w}}^{2}+\frac{1}{6}\left(\bar{w}-\bar{z}^{\prime}\right) \partial_{\bar{w}}^{3}\right) \int d^{2} z \frac{1}{(\bar{z}-\bar{w})\left(z-z^{\prime}\right)} \\
& =\frac{\pi}{6} \frac{1}{\left(\bar{w}-\bar{z}^{\prime}\right)^{2}},
\end{aligned}
$$

where in the last equality we used (B.8). This agrees with the general formula (B.17).

The second integral of our interest is

$$
\begin{aligned}
I_{2}^{(0,-4)}\left(w, z^{\prime}\right) & =\int d^{2} z \frac{1}{(\bar{z}-\bar{w})^{4}} \frac{\left(\bar{z}-\bar{z}^{\prime}\right)^{2}}{z-z^{\prime}}=\frac{1}{6} \partial_{\bar{w}}^{3} \int d^{2} z \frac{1}{(\bar{z}-\bar{w})} \frac{\left(\bar{z}-\bar{z}^{\prime}\right)^{2}}{z-z^{\prime}} \\
& =\frac{1}{6} \partial_{\bar{w}}^{3} \int d^{2} z \frac{(\bar{z}-\bar{\eta})^{2}}{(z-\eta) \bar{z}}, \quad \eta=z^{\prime}-w \\
& =\frac{1}{6} \partial_{\bar{w}}^{3} \int d^{2} z\left[\frac{\bar{z}}{z-\eta}-\frac{2 \eta}{z-\eta}+\bar{\eta}^{2} \frac{1}{\bar{z}(z-\eta)}\right] \\
& =-\frac{1}{6} \partial_{\bar{\eta}}^{3} \int d^{2} z\left[\frac{\bar{z}+\bar{\eta}}{z}-\frac{2 \eta}{z}+\bar{\eta}^{2} \frac{1}{\bar{z}(z-\eta)}\right] \\
& =\frac{\pi}{3} \frac{1}{\bar{z}^{\prime}-\bar{w}},
\end{aligned}
$$

where we used the translational invariance several times and (B.8) in the last equality. This is again consistent with the general formula (B.17). One can also verify the relation $\partial_{\bar{z}^{\prime}} I_{2}^{(0,-4)}\left(w, z^{\prime}\right)=-2 I_{1}^{(0,-4)}\left(w, z^{\prime}\right)$ as expected. These two examples $I_{1}^{(0,-4)}, I_{2}^{(0,-4)}$ are directly related to the derivation of stress tensor Ward identity in (5.7).

Therefore we have checked our general formula (B.17) in various examples.

\section{General celestial OPEs in EFT}

We will consider the collinear limit in effective field theory. In particular, we consider the collinear limit arising from the cubic vertex involving massless particles with helicity 
$s_{1}, s_{2}, s_{3}$. In this limit, the amplitude satisfies

$$
A_{n}\left(1^{s_{1}}, 2^{s_{2}}, \cdots\right) \stackrel{p_{1} / / p_{2}}{\longrightarrow} \sum_{s_{3}} \operatorname{Split}\left(1^{s_{1}}+2^{s_{2}} \rightarrow P^{-s_{3}}\right) A_{n-1}\left(P^{-s_{3}}, \cdots\right),
$$

where $P=p_{1}+p_{2}$ and the dots represent the rest of $n-2$ particles which are not relevant here. In the collinear limit, the scattering amplitude is also supposed to factorize as follows:

$$
A_{n}\left(1^{s_{1}}, 2^{s_{2}}, \cdots\right) \stackrel{p_{1} / / p_{2}}{\longrightarrow} \sum_{s_{3}} A_{3}\left(1^{s_{1}}+2^{s_{2}},-P^{s_{3}}\right) \frac{1}{P^{2}} A_{n-1}\left(P^{-s_{3}}, \cdots\right) .
$$

Therefore we have

$$
\text { Split }\left(1^{s_{1}}+2^{s_{2}} \rightarrow P^{-s_{3}}\right)=A_{3}\left(1^{s_{1}}, 2^{s_{2}},-P^{s_{3}}\right) \frac{1}{P^{2}} \propto \frac{1}{\langle 12\rangle[12]}[12]^{s-2 s_{3}}[1 P]^{s-2 s_{2}}[P 2]^{s-2 s_{1}},
$$

where we use the property that the three-point amplitude $A_{3}$ is uniquely fixed by the symmetry and locality [43]. Also we assume that $s \equiv s_{1}+s_{2}+s_{3} \geq 0$, so locality guarantees that only square brackets appear in the three-point amplitude. And $P^{2}=\left(p_{1}+p_{2}\right)^{2}=$ $2 p_{1} \cdot p_{2} \propto\langle 12\rangle[12]$. Note that we will not keep track of any overall constant as they can be absorbed into the effective coupling which we also don't write down explicitly.

Because of momentum conversation $P=p_{1}+p_{2}$, we actually have (for $\left.s \geq 0\right)^{30}$

$$
\begin{aligned}
\langle i j\rangle & =0 & & \rightarrow & \lambda_{P} & =\alpha_{1} \lambda_{1}=\alpha_{2} \lambda_{2}, \\
P & =p_{1}+p_{2} & & \rightarrow & \lambda_{P} \tilde{\lambda}_{P} & =\lambda_{1} \tilde{\lambda}_{1}+\lambda_{2} \tilde{\lambda}_{2} .
\end{aligned}
$$

This gives

$$
\tilde{\lambda}_{P}=\frac{1}{\alpha_{1}} \tilde{\lambda}_{1}+\frac{1}{\alpha_{2}} \tilde{\lambda}_{2}
$$

and thus

$$
[1 P]=\frac{1}{\alpha_{2}}[12], \quad[P 2]=\frac{1}{\alpha_{1}}[12] .
$$

Furthermore for physical momentum $\tilde{\lambda}=\lambda^{*}$, we thus have $1 / \alpha_{1}^{2}+1 / \alpha_{2}^{2}=1$ which enables us to set $1 / \alpha_{1}=\sqrt{x}, 1 / \alpha_{2}=\sqrt{1-x}$. As a consequence, $p_{1}=x P, p_{2}=(1-x) P$ and $\omega_{1}=x \omega_{P}, \omega_{2}=(1-x) \omega_{P} .{ }^{31}$

With these relation, the split factor can be evaluated explicitly

$$
\begin{aligned}
\operatorname{Split}\left(1^{s_{1}}+2^{s_{2}} \rightarrow P^{-s_{3}}\right) & \propto \frac{[12]^{s-1}}{\langle 12\rangle}\left(\frac{[1 P]}{[12]}\right)^{s-2 s_{2}}\left(\frac{[P 2]}{[12]}\right)^{s-2 s_{1}} \\
& =\frac{[12]^{s-1}}{\langle 12\rangle}(\sqrt{x})^{s-2 s_{2}}(\sqrt{1-x})^{s-2 s_{1}} \\
& \propto \frac{\bar{z}_{12}^{s-1}}{z_{12}} \omega_{1}^{s_{2}+s_{3}-1} \omega_{2}^{s_{1}+s_{3}-1} \omega_{P}^{-s_{3}}
\end{aligned}
$$

\footnotetext{
${ }^{30}$ To be more rigorous, one needs to deviate from the strict collinear limit a little bit. Since we are only interested in the leading singular term, this infinitesimal deviation is not important.

${ }^{31}$ Here $\omega$ is the energy along the null direction such that $p_{i}^{\mu}=\eta_{i} \omega_{i} q_{i}^{\mu}$ and $q=(1+z \bar{z}, z+\bar{z},-i(z-\bar{z}), 1-z \bar{z})$. In the collinear limit, $q_{i}$ 's are approximately the same.
} 
where we used $\langle i j\rangle=-2 \sqrt{\omega_{i} \omega_{j}} z_{i j},[i j]=2 \eta_{i} \eta_{j} \sqrt{\omega_{i} \omega_{j}} \bar{z}_{i j}, z_{i j}=z_{i}-z_{j}, \bar{z}_{i j}=\bar{z}_{i}-\bar{z}_{j}$ and $\eta_{i}= \pm 1$ distinguishes outgoing/incoming particles.

We are however interested in the celestial amplitude which is defined as

$$
\mathcal{M}_{n}\left(\Delta_{i}, J_{i}, z_{i}, \bar{z}_{i}\right)=\left(\prod_{j=1}^{n} \int_{0}^{\infty} d \omega_{j} \omega_{j}^{\Delta_{j}-1}\right) \mathcal{A}_{n}\left(J_{i}, p_{i}^{\mu}\right)
$$

where $J_{i}$ is the spin of the operator in 2 d, while in $4 \mathrm{~d}$ it is the helicity of the particle, namely $J_{i}=s_{i}$. The celestial amplitude can be regarded as a conformal correlator on the celestial sphere

$$
\mathcal{M}_{n}\left(\Delta_{i}, J_{i}, z_{i}, \bar{z}_{i}\right)=\left\langle\mathcal{O}_{\Delta_{1}, J_{1}}^{\eta_{1}}\left(z_{1}, \bar{z}_{1}\right) \cdots \mathcal{O}_{\Delta_{n}, J_{n}}^{\eta_{n}}\left(z_{n}, \bar{z}_{n}\right)\right\rangle
$$

To finally obtain the OPE, we just need to perform the Mellin transformation for (C.10). In particular, we have the Mellin transformation of $\omega_{1}^{\alpha} \omega_{2}^{\beta}\left(\omega_{1}+\omega_{2}\right)^{\gamma}$ in the split factor:

$\int_{0}^{\infty} d \omega_{2} \omega_{2}^{\Delta_{2}-1} \int_{0}^{\infty} d \omega_{1} \omega_{1}^{\Delta_{1}-1} \omega_{1}^{\alpha} \omega_{2}^{\beta}\left(\omega_{1}+\omega_{2}\right)^{\gamma} f\left(\omega_{1}+\omega_{2}\right)=B\left(\Delta_{1}+\alpha, \Delta_{2}+\beta\right) \int_{0}^{\infty} d \omega \omega^{\Delta_{P}-1} f(\omega)$,

where we used (2.15) and $\Delta_{P}=\Delta_{1}+\Delta_{2}+\alpha+\beta+\gamma$.

With this formula, we finally obtain tree level leading order celestial OPE arsing from cubic vertex in EFT:

$$
\mathcal{O}_{\Delta_{1}, J_{1}}\left(z_{1}, \bar{z}_{1}\right) \mathcal{O}_{\Delta_{2}, J_{2}}\left(z_{2}, \bar{z}_{2}\right) \sim \frac{\bar{z}_{12}^{J_{1}+J_{2}+J_{3}-1}}{z_{12}} B\left(\Delta_{1}+J_{2}+J_{3}-1, \Delta_{2}+J_{1}+J_{3}-1\right) \mathcal{O}_{\Delta_{3},-J_{3}}\left(z_{2}, \bar{z}_{2}\right),
$$

where $\Delta_{3}=\Delta_{1}+\Delta_{2}+J_{1}+J_{2}+J_{3}-2$.

\section{Soft photon theorems with magnetic corrections}

The leading and sub-leading soft photon theorems state that

$$
\lim _{p \rightarrow 0} M_{n+1}\left(p^{s}, p_{1}, p_{2}, \cdots, p_{n}\right)=\left(S_{s}^{(0)}+S_{s}^{(1)}+\cdots\right) M_{n}\left(p_{1}, p_{2}, \cdots p_{n}\right),
$$

with soft factors

$$
S_{s}^{(0)}=\sum_{k=1}^{n} \eta_{k} \frac{\left(e_{k} \epsilon_{s}+g_{k} \tilde{\epsilon}_{s}\right) \cdot p_{k}}{p \cdot p_{k}}, \quad S_{s}^{(1)}=\sum_{k=1}^{n} i \eta_{k} \frac{\left(e_{k} \epsilon_{s}^{\mu}+g_{k} \tilde{\epsilon}_{s}^{\mu}\right) p^{\nu} J_{k \mu \nu}}{p \cdot p_{k}}
$$

where $\eta_{k}= \pm 1$ for out-going/in-coming particle, $s$ is the helicity of the soft photon, $e_{k}, g_{k}$ are the electric and magnetic charges of $k$-th matter particle, while $q_{k}^{\mu}, J_{k \mu \nu}$ represent momentum and angular momentum. In the absence of magnetic charge, these are the Weinberg's soft photon theorem and Low's sub-leading soft photon theorem. The role of magnetic charges can be incorporated through the electro-magnetic duality transformation, which can be regarded as a phase rotation of couplings. The invariance of soft factors under the gauge transformation $\epsilon_{s}^{\mu} \rightarrow \epsilon_{s}^{\mu}+p^{\mu}, \tilde{\epsilon}_{s}^{\mu} \rightarrow \tilde{\epsilon}_{s}^{\mu}+p^{\mu}$ is equivalent to the conservation of electric and magnetic charges. 
It is convenient to parametrize the momentum in terms of celestial coordinates as follows:

$$
p^{\mu}=\omega q^{\mu}, \quad q^{\mu}=(1+z \bar{z}, \quad z+\bar{z}, \quad-i(z-\bar{z}), \quad 1-z \bar{z}) .
$$

Then the (electric) polarization vectors can be chosen as:

$$
\epsilon_{+}^{\mu}(p)=\frac{1}{\sqrt{2}} \partial_{z} q^{\mu}=(\bar{z}, 1,-i,-\bar{z}), \quad \epsilon_{-}^{\mu}(p)=\frac{1}{\sqrt{2}} \partial_{\bar{z}} q^{\mu}=(z, 1, i,-z),
$$

satisfying

$$
\epsilon_{+} \cdot p=\epsilon_{-} \cdot p=0, \quad \epsilon_{+} \cdot \epsilon_{+}=\epsilon_{-} \cdot \epsilon_{-}=0, \quad \epsilon_{+} \cdot \epsilon_{-}=1 .
$$

We also need the magnetic polarization vectors which are defined as follows [44]

$$
\tilde{\epsilon}_{ \pm \mu}(p)=\frac{\varepsilon_{\mu \nu \rho \sigma} n^{\nu} p^{\rho} \epsilon_{ \pm}^{\sigma}(p)}{p \cdot n}
$$

where $n^{\rho}$ is an arbitrary reference vector. Explicit computations show that

$$
\tilde{\epsilon}_{+}^{\mu}(p)=i \epsilon_{+}^{\mu}(p)+r p^{\mu}, \quad \tilde{\epsilon}_{-}^{\mu}(p)=-i \epsilon_{-}^{\mu}(p)+\bar{r} p^{\mu},
$$

where $r$ is function of $n^{\mu}$ and $p^{\mu}$. Since $r p^{\mu}, \bar{r} p^{\mu}$ just correspond to gauge transformations, they can be dropped out in the polarizations. As a result, the on-shell amplitudes do not depend on the reference vector $n^{\mu}$. The magnetic polarizations satisfy the same relation as the electric ones in (D.5).

Using celestial coordinates, the soft factors for positive soft photon are given by

$$
S_{+}^{(0)}=\sum_{k=1}^{n} \eta_{k} \frac{Q_{k}}{\omega\left(z-z_{k}\right)}, \quad S_{+}^{(1)}=\sum_{k=1}^{n} \eta_{k} \frac{Q_{k}}{z-z_{k}}\left(\frac{J_{k}}{\omega_{k}}+\partial_{\omega_{k}}+\frac{\bar{z}-\bar{z}_{k}}{\omega_{k}} \bar{\partial}_{k}\right), \quad Q_{k}=e_{k}+i g_{k} .
$$

For negative soft photon, the soft factors are given by its complex conjugate.

Open Access. This article is distributed under the terms of the Creative Commons Attribution License (CC-BY 4.0), which permits any use, distribution and reproduction in any medium, provided the original author(s) and source are credited.

\section{References}

[1] H. Bondi, M.G.J. van der Burg and A.W.K. Metzner, Gravitational waves in general relativity. 7. Waves from axisymmetric isolated systems, Proc. Roy. Soc. Lond. A 269 (1962) 21 [INSPIRE].

[2] R.K. Sachs, Gravitational waves in general relativity. 8. Waves in asymptotically flat space-times, Proc. Roy. Soc. Lond. A 270 (1962) 103 [INSPIRE].

[3] A. Strominger, On BMS Invariance of Gravitational Scattering, JHEP 07 (2014) 152 [arXiv: 1312.2229] [INSPIRE].

[4] T. He, V. Lysov, P. Mitra and A. Strominger, BMS supertranslations and Weinberg's soft graviton theorem, JHEP 05 (2015) 151 [arXiv:1401.7026] [INSPIRE]. 
[5] A. Strominger, Lectures on the Infrared Structure of Gravity and Gauge Theory, arXiv: 1703.05448 [INSPIRE].

[6] C. Cheung, A. de la Fuente and R. Sundrum, $4 D$ scattering amplitudes and asymptotic symmetries from $2 D$ CFT, JHEP 01 (2017) 112 [arXiv:1609.00732] [INSPIRE].

[7] S. Pasterski, S.-H. Shao and A. Strominger, Flat Space Amplitudes and Conformal Symmetry of the Celestial Sphere, Phys. Rev. D 96 (2017) 065026 [arXiv:1701.00049] [inSPIRE].

[8] S. Pasterski and S.-H. Shao, Conformal basis for flat space amplitudes, Phys. Rev. D 96 (2017) 065022 [arXiv: 1705.01027] [INSPIRE].

[9] A.-M. Raclariu, Lectures on Celestial Holography, arXiv:2107.02075 [INSPIRE].

[10] S. Pasterski, Lectures on celestial amplitudes, Eur. Phys. J. C 81 (2021) 1062 [arXiv: 2108.04801] [INSPIRE].

[11] A. Guevara, E. Himwich, M. Pate and A. Strominger, Holographic symmetry algebras for gauge theory and gravity, JHEP 11 (2021) 152 [arXiv:2103.03961] [INSPIRE].

[12] A. Strominger, $w_{1+\infty}$ and the Celestial Sphere, arXiv:2105.14346 [INSPIRE].

[13] S. Banerjee, S. Ghosh and P. Paul, MHV graviton scattering amplitudes and current algebra on the celestial sphere, JHEP 02 (2021) 176 [arXiv: 2008.04330] [INSPIRE].

[14] S. Banerjee and S. Ghosh, MHV gluon scattering amplitudes from celestial current algebras, JHEP 10 (2021) 111 [arXiv:2011.00017] [InSPIRE].

[15] S. Pasterski, A. Puhm and E. Trevisani, Celestial diamonds: conformal multiplets in celestial CFT, JHEP 11 (2021) 072 [arXiv:2105.03516] [INSPIRE].

[16] T. He, P. Mitra and A. Strominger, 2D Kac-Moody Symmetry of 4D Yang-Mills Theory, JHEP 10 (2016) 137 [arXiv: 1503. 02663] [INSPIRE].

[17] M. Pate, A.-M. Raclariu, A. Strominger and E.Y. Yuan, Celestial operator products of gluons and gravitons, Rev. Math. Phys. 33 (2021) 2140003 [arXiv:1910.07424] [INSPIRE].

[18] H. Elvang, C.R.T. Jones and S.G. Naculich, Soft Photon and Graviton Theorems in Effective Field Theory, Phys. Rev. Lett. 118 (2017) 231601 [arXiv:1611.07534] [InSPIRE].

[19] E. Himwich, M. Pate and K. Singh, Celestial Operator Product Expansions and $\mathrm{w}_{1+\infty}$ Symmetry for All Spins, arXiv:2108.07763 [INSPIRE].

[20] W. Fan, A. Fotopoulos and T.R. Taylor, Soft Limits of Yang-Mills Amplitudes and Conformal Correlators, JHEP 05 (2019) 121 [arXiv:1903.01676] [INSPIRE].

[21] A. Fotopoulos, S. Stieberger, T.R. Taylor and B. Zhu, Extended Super BMS Algebra of Celestial CFT, JHEP 09 (2020) 198 [arXiv:2007.03785] [INSPIRE].

[22] H. Jiang, Celestial superamplitude in $\mathcal{N}=4$ SYM theory, JHEP 08 (2021) 031 [arXiv:2105.10269] [INSPIRE].

[23] B. Czech, L. Lamprou, S. McCandlish, B. Mosk and J. Sully, A Stereoscopic Look into the Bulk, JHEP 07 (2016) 129 [arXiv: 1604.03110] [INSPIRE].

[24] P. Kravchuk and D. Simmons-Duffin, Light-ray operators in conformal field theory, JHEP 11 (2018) 102 [arXiv: 1805.00098] [INSPIRE].

[25] P. Di Francesco, P. Mathieu and D. Senechal, Conformal Field Theory, Graduate Texts in Contemporary Physics, Springer-Verlag, New York (1997) [DOI] [INSPIRE]. 
[26] W. Fan, A. Fotopoulos, S. Stieberger and T.R. Taylor, On Sugawara construction on Celestial Sphere, JHEP 09 (2020) 139 [arXiv: 2005.10666] [INSPIRE].

[27] A. Puhm, Conformally Soft Theorem in Gravity, JHEP 09 (2020) 130 [arXiv:1905.09799] [INSPIRE].

[28] T. Adamo, L. Mason and A. Sharma, Celestial amplitudes and conformal soft theorems, Class. Quant. Grav. 36 (2019) 205018 [arXiv: 1905.09224] [INSPIRE].

[29] A. Guevara, Notes on Conformal Soft Theorems and Recursion Relations in Gravity, arXiv: 1906.07810 [INSPIRE].

[30] M. Pate, A.-M. Raclariu and A. Strominger, Conformally Soft Theorem in Gauge Theory, Phys. Rev. D 100 (2019) 085017 [arXiv:1904.10831] [INSPIRE].

[31] D. Nandan, A. Schreiber, A. Volovich and M. Zlotnikov, Celestial Amplitudes: Conformal Partial Waves and Soft Limits, JHEP 10 (2019) 018 [arXiv:1904.10940] [INSPIRE].

[32] A. Nande, M. Pate and A. Strominger, Soft Factorization in QED from 2D Kac-Moody Symmetry, JHEP 02 (2018) 079 [arXiv: 1705. 00608] [INSPIRE].

[33] E. Himwich and A. Strominger, Celestial current algebra from Low's subleading soft theorem, Phys. Rev. D 100 (2019) 065001 [arXiv:1901.01622] [INSPIRE].

[34] A. Strominger, Magnetic Corrections to the Soft Photon Theorem, Phys. Rev. Lett. 116 (2016) 031602 [arXiv: 1509.00543] [INSPIRE].

[35] H. Osborn, Conformal Blocks for Arbitrary Spins in Two Dimensions, Phys. Lett. B 718 (2012) 169 [arXiv: 1205.1941] [INSPIRE].

[36] D. Kapec, P. Mitra, A.-M. Raclariu and A. Strominger, 2D Stress Tensor for $4 D$ Gravity, Phys. Rev. Lett. 119 (2017) 121601 [arXiv:1609.00282] [INSPIRE].

[37] A. Fotopoulos and T.R. Taylor, Primary Fields in Celestial CFT, JHEP 10 (2019) 167 [arXiv:1906.10149] [INSPIRE].

[38] Y. Hamada and G. Shiu, Infinite Set of Soft Theorems in Gauge-Gravity Theories as Ward-Takahashi Identities, Phys. Rev. Lett. 120 (2018) 201601 [arXiv:1801.05528] [InSPIRE].

[39] Z.-Z. Li, H.-H. Lin and S.-Q. Zhang, Infinite Soft Theorems from Gauge Symmetry, Phys. Rev. D 98 (2018) 045004 [arXiv: 1802.03148] [INSPIRE].

[40] D. Kapec and P. Mitra, A d-Dimensional Stress Tensor for Mink ${ }_{d+2}$ Gravity, JHEP 05 (2018) 186 [arXiv: 1711.04371$]$ [INSPIRE].

[41] Z. Bern, S. Davies and J. Nohle, On Loop Corrections to Subleading Soft Behavior of Gluons and Gravitons, Phys. Rev. D 90 (2014) 085015 [arXiv:1405.1015] [INSPIRE].

[42] T. He, D. Kapec, A.-M. Raclariu and A. Strominger, Loop-Corrected Virasoro Symmetry of 4D Quantum Gravity, JHEP 08 (2017) 050 [arXiv: 1701.00496] [INSPIRE].

[43] H. Elvang and Y.-t. Huang, Scattering Amplitudes, arXiv:1308.1697 [INSPIRE].

[44] J. Terning and C.B. Verhaaren, Resolving the Weinberg Paradox with Topology, JHEP 03 (2019) 177 [arXiv: 1809.05102] [INSPIRE]. 